\title{
Counting invertible Schrödinger Operators over Finite Fields for Trees, Cycles and Complete Graphs
}

\author{
Roland Bacher* \\ Institut Fourier (CNRS UMR 5582) \\ Univ. Grenoble Alpes \\ 38000 Grenoble, France \\ Roland.Bacher@ujf-grenoble.fr
}

Submitted: Apr 13, 2015; Accepted: Dec 2, 2015; Published: Dec 11, 2015

Mathematics Subject Classifications: 05C31, 05C10, 20G40

\begin{abstract}
We count invertible Schrödinger operators (perturbations by diagonal matrices of the adjacency matrix) over finite fields for trees, cycles and complete graphs. This is achieved for trees through the definition and use of local invariants (algebraic constructions of perhaps independent interest). Cycles and complete graphs are treated by ad hoc methods.
\end{abstract}

Keywords: tree; graph; enumerative combinatorics; invariants; Schrödinger operator

\section{Introduction}

A Schrödinger operator (or perhaps more accurately, an opposite of a Schrödinger operator) on a graph $G$ (always finite with unoriented edges and no loops or multiple edges) is a matrix obtained by adding an arbitrary diagonal matrix to the adjacency matrix of $G$.

Our first result counts invertible Schrödinger operators over finite fields for trees (graphs without closed non-trivial paths):

Theorem 1. The number of invertible Schrödinger operators of a finite tree $T$ with $n$ vertices over the finite field $\mathbb{F}_{q}$ is given by

$$
(-\sqrt{-q})^{n} \chi_{T}(\sqrt{-q}+1 / \sqrt{-q})
$$

where $\chi_{T}=\operatorname{det}\left(x I_{n}-A\right) \in \mathbb{Z}[x]$ is the characteristic polynomial of the adjacency matrix A of $T$.

\footnotetext{
*Partially supported by the LabEx PERSYVAL-Lab (ANR-11-LABX-0025). The author is a member of the project-team GALOIS supported by this LabEx.
} 
Theorem 1 is wrong for arbitrary graphs: It fails to yield integral evaluations at prime-powers for non-bipartite graphs. It is also wrong for bipartite graphs: Formula (1) amounts to $q^{4}-2 q^{2}+1$ for the the 4 -cycle $C_{4}$ which has $(q-1)\left(q^{3}-q-1\right)=q^{4}-q^{3}-q^{2}+1$ invertible Schrödinger operators over $\mathbb{F}_{q}$ by Theorem 2 .

The key-ingredient for proving Theorem 1 is the notion of local invariants, a general framework for computing invariants of finite (plane) trees.

Our next result enumerates invertible Schrödinger operators for the $n$-cycle $C_{n}$ defined as the unique connected graph consisting of $n \geqslant 3$ vertices of degree 2 :

Theorem 2. The number $S_{C_{n}}$ of invertible Schrödinger operators for the $n$-cycle $C_{n}$ over $\mathbb{F}_{q}$ is given by

$$
\begin{aligned}
S_{C_{2 n+1}} & =q^{2 n+1}-\frac{1-q^{2 n+2}}{1-q^{2}}, \\
S_{C_{4 n}} & =q^{4 n}-q^{2 n}+\frac{\left(1-q^{2 n}\right)\left(1-q^{2 n+1}\right)}{1-q^{2}}, \\
S_{C_{4 n+2}} & =q^{4 n+2}+\frac{\left(1-q^{2 n+1}\right)\left(1-q^{2 n+2}\right)}{1-q^{2}} .
\end{aligned}
$$

if $q$ is odd and by

$$
\begin{aligned}
S_{C_{2 n+1}} & =q^{2 n+1}-\frac{1-q^{2 n+2}}{1-q^{2}}, \\
S_{C_{2 n}} & =q^{2 n}-q^{n}+\frac{\left(1-q^{n}\right)\left(1-q^{n+1}\right)}{1-q^{2}}
\end{aligned}
$$

if $q$ is even.

Observe that $S_{C_{n}}$ is polynomial in $q$, except if $n \equiv 2(\bmod 4)$ where it is given by two polynomials, depending on the parity of $q$.

The proof of Theorem 2 is essentially an identity in $\mathbb{Z}\left[\mathrm{SL}_{2}\left(\mathbb{F}_{q}\right)\right]$, see Theorem 18 . This identity is of independent interest: It yields for example Bose-Mesner algebras and a good generator of random elements in $\mathrm{SL}_{2}\left(\mathbb{F}_{q}\right)$.

Invertible Schrödinger operators for the complete graph $K_{n}$ on $n$ vertices are invertible matrices of size $n \times n$ with arbitrary diagonal coefficients and with all off-diagonal coefficients equal to 1 . The following result gives their number over finite fields:

Theorem 3. The number of invertible Schrödinger operators over $\mathbb{F}_{q}$ associated to the complete graph on $n$ vertices is given by

$$
\frac{(q-1)^{n+1}+(-1)^{n}}{q}+n(q-1)^{n-1} .
$$

The content of the paper is organized as follows:

Section 2 introduces and gives examples of local invariants, the main tool for proving Theorem 1, established in Section 3. 
Section 4 describes a few additional properties of the polynomial $S_{T}(q)$ enumerating invertible Schrödinger operators over $\mathbb{F}_{q}$ of a finite tree $T$.

Section 5 extends local invariants to trees having coloured vertices.

Section 6 studies the behaviour of the polynomial $S_{T}(q)$ (defined by Theorem 1) under edge-subdivisions.

Section 7 refines $S_{T}$ in order to count invertible Schrödinger operators (of trees) over finite fields according to multiplicities of values of the Jacobi symbol on the diagonal.

In Section 8 we give formulae for the coefficients of

$$
\left(\sum_{\mu \in M} \sum_{x \in \mathbb{F}_{q}}\left[\left(\begin{array}{cc}
x & \mu \\
-1 / \mu & 0
\end{array}\right)\right]\right)^{n} \in \mathbb{Z}\left[\mathrm{SL}_{2}\left(\mathbb{F}_{q}\right)\right]
$$

where $M$ is a subgroup of the multiplicative group $\mathbb{F}_{q}^{*}$ of units in $\mathbb{F}_{q}$. Theorem 2 is a rather straightforward consequence of these formulae, as shown in Section 9.

Section 10 gives an easy proof of Theorem 3 .

A short last Section 11 contains a few final remarks.

\section{Local invariants}

\section{$2.1 \quad$ Local construction of trees}

We denote by $\mathcal{T}$ the set of all finite trees and by $\mathcal{R}$ the set of all finite rooted trees.

Every element of $\mathcal{R}$ can be constructed (generally not uniquely) in a finite number of steps involving the following operations:

$(\boldsymbol{V})$ Creating a trivial rooted tree consisting of a unique root-vertex.

$(\boldsymbol{E})$ Extending a rooted tree by gluing one end of an additional edge to the root-vertex and by moving the root vertex to the other end of the newly attached edge.

$(\boldsymbol{M})$ Merging two rooted trees by gluing their root-vertices into the root-vertex of the resulting tree.

The operation $E$ increases the number of edges and vertices by 1 . The operation $M$, applied to two rooted trees having respectively $a$ and $b$ vertices produces a rooted tree with $a+b-1$ vertices and $a+b-2$ edges.

$V$ is constant (independent of any arguments), $E$ operates on elements of $\mathcal{R}$. The map $M$ defines a commutative and associative product which turns $\mathcal{R}$ into a commutative monoid with identity $V$ representing the trivial rooted tree reduced to the root vertex. The monoid $(\mathcal{R}, M)$ is $\mathbb{N}$-graded: The degree of a rooted tree $R$ is the number of nonroot vertices in $R$. The sum over all possible contractions of an edge starting at the root vertex defines a derivation of degree -1 on the graded monoid-ring $\mathbb{K}[\mathcal{R}]$ (over a commutative ring or field $\mathbb{K}$ ). The map $E$ can thus be thought of as an "integral operator" on $\mathcal{R}$. Algebraically, $\mathbb{K}[\mathcal{R}]$ is the free commutative algebra with generators 
$\{E(R)\}_{R \in \mathcal{R}}$. Its Hilbert series $\sum_{n=0}^{\infty} \alpha_{n} x^{n}$ encoding the dimension $\alpha_{n}$ of homogeneous polynomials of degree $n$ in $\mathbb{K}[\mathcal{R}]$ satisfies the identity

$$
\sum_{n=0}^{\infty} \alpha_{n} x^{n}=\prod_{n=0}^{\infty}\left(\frac{1}{1-x^{n+1}}\right)^{\alpha_{n}}
$$

appearing already in [1], and starts as $1,1,2,4,9,20,48,115,286$, see sequence A81 of [3].

Finally, the "forget" operator,

$(\boldsymbol{F})$ Forgetting the root-structure by turning the root of a rooted tree into an ordinary vertex,

induces a surjection from $\mathcal{R}$ onto $\mathcal{T}$. We have the identity

$$
F(M(A, E(B)))=F(M(E(A), B))
$$

(for all $A, B$ in $\mathcal{R}$ ) mirroring the fact that an ordinary tree with $n$ vertices can be rooted at $n$ different vertices. Identity (3) amounts to requiring $(A, B) \longmapsto F(M(A, E(B)))$ to be symmetric in its arguments $A$ and $B$.

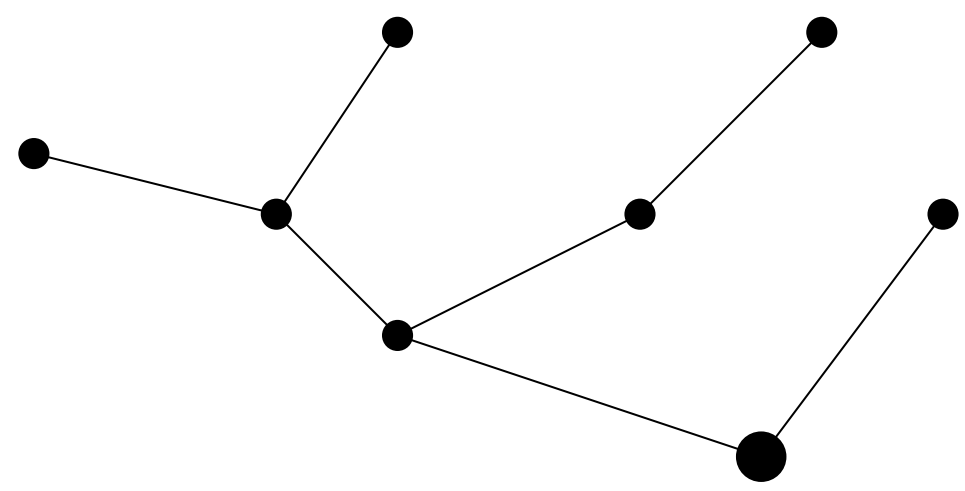

Figure 1: An example of a tree, rooted at the largest dot.

Figure 1 shows the rooted tree encoded (for example) by

$$
M\{E(M\{E(M\{E(V), E(V)\}), E(E(V))\}), E(V)\}
$$

with curly brackets enclosing arguments of $M$.

\subsection{Digression: plane trees}

A plane tree is a tree embedded in the oriented plane, up to orientation-preserving homeomorphisms. Plane trees are abstract trees together with cyclic orders on sets of edges sharing a common vertex.

A rooted plane tree is a plane tree having a root together with a refinement into a linear order of the cyclic order on root-edges containing the root-vertex. 
Rooted plane trees can be constructed using the operators $V, E, M$ already considered, except that the associative product $M$ is no longer commutative. The operator $F$ turning the root-vertex into an ordinary vertex satisfies (3) and the "trace-identity"

$$
F(M(A, B))=F(M(B, A)) .
$$

The set $\Pi$ of all finite rooted plane trees is a non-commutative monoid. It is again graded (by the number of non-root vertices) and $\mathbb{K}[\Pi]$ is a non-commutative differential algebra. Algebraically, $\mathbb{K}[\Pi]$ is the free non-commutative algebra with generators

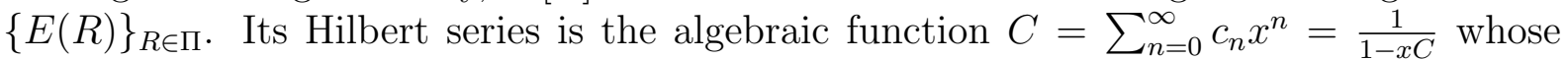
coefficients define the famous sequence $1,1,2,5,14, \ldots, c_{n}=\left(\begin{array}{c}2 n \\ n\end{array}\right) \frac{1}{n+1}$ of Catalan numbers, see sequence A108 of [3].

\subsection{Local invariants of (rooted) trees}

A local invariant of rooted trees with values in a commutative monoid $\mathbb{E}$ is a map $i$ : $\mathcal{R} \longrightarrow \mathbb{E}$ which can be computed by replacing the construction operators $V, E, M$ by $v, e, m$ where $v=1$ is the multiplicative identity of $\mathbb{E}$, where $e: \mathbb{E} \longrightarrow \mathbb{E}$ is an arbitrary map and where $m: \mathbb{E} \times \mathbb{E} \longrightarrow \mathbb{E}$ is the product of the monoid $\mathbb{E}$.

A local invariant of trees is a map $f \circ i$ from $\mathcal{T}$ to a set of values $\mathbb{F}$ where $i$ is a local invariant of rooted trees given by maps $v, e, m: \mathbb{E}^{*} \longrightarrow \mathbb{E}$ as above (with $\mathbb{E}^{*}$ denoting respectively $\emptyset, \mathbb{E}$ and $\mathbb{E}^{2}$ ) and where $f: \mathbb{E} \longrightarrow \mathbb{F}$ satisfies the identity

$$
f(m(A, e(B)))=f(m(e(A), B))
$$

corresponding to (3) for all $A, B$ in $\mathbb{E}$.

A trivial example with $\mathbb{E}=\mathbb{F}=\mathbb{N}$ is given by $v=0, e(x)=x+1, m(x, y)=x+y$ and $f(x)=x$. It counts the number of edges (given by $n-1$ for a tree with $n$ vertices) of a tree. Replacing $f$ with $f_{1}(x)=x+1$ we count vertices instead of edges.

Remark 4. The definition of local invariants for (rooted) trees is tautological: Every map $A: \mathcal{T} \longrightarrow \mathbb{F}$ is a local invariant on the set $\mathcal{T}$ of all finite trees by taking $\mathbb{E}=\mathcal{R}$ and $v=V, e=E, m=M, f=A \circ F$. We are of course interested in local invariants where the maps $v, e, m$ and $f$ are simple, e.g. given by algebraic operations over some commutative monoid $\mathbb{E}$ with a rich algebraic structure.

The terminology "local" alludes to the fact that local invariants can be computed algorithmically using "local" operations which modify only neighbourhoods of root-vertices.

\subsection{Examples of local invariants}

\subsubsection{Enumeration of (maximal) independent sets}

A subset $\mathcal{I}$ of vertices in a graph $G$ is independent if two distinct elements of $\mathcal{I}$ are never adjacent. The polynomial $\sum_{j} \alpha_{j} x^{j}$ encoding the number $\alpha_{j}$ of independent sets with $j$ vertices in a finite tree can be computed as a local invariant using

$$
v=(1, x),
$$




$$
\begin{aligned}
e(a, b) & =(a+b, x a), \\
m((a, b),(\alpha, \beta)) & =\left(a \alpha, \frac{1}{x} b \beta\right), \\
f(a, b) & =a+b .
\end{aligned}
$$

We leave the easy details to the reader. (Hint: The first coefficient $a$ of $(a, b)$ counts independent sets without the root of rooted trees, the second coefficient $b$ counts independent sets containing the root-vertex.)

For the tree underlying Figure 1 we get

$$
1+8 x+21 x^{2}+22 x^{3}+8 x^{4}+x^{5} .
$$

An independent set $\mathcal{I}$ of a graph $G$ is maximal if every vertex of $G$ is at most at distance 1 to $\mathcal{I}$ (i.e. a vertex $v$ is either in $\mathcal{I}$ or adjacent to an element of $\mathcal{I}$ ).

The polynomial $\sum_{j} \beta_{j} x^{j}$ encoding the number $\beta_{j}$ of maximal independent sets with $j$ vertices in a finite tree can be computed as a local invariant using

$$
\begin{aligned}
v & =(1,0, x), \\
e(a, b, c) & =(b, c, x(a+b)), \\
m((a, b, c),(\alpha, \beta, \gamma)) & =\left(a \alpha, a \beta+b \alpha+b \beta, \frac{1}{x} c \gamma\right), \\
f(a, b, c) & =b+c .
\end{aligned}
$$

( $a$ encodes non-maximal independent sets $\mathcal{I}$ not containing the root $r$ of a rooted tree $R$ such that $\mathcal{I} \cup\{r\}$ is maximal independent in $R$ and $\mathcal{I}$ is maximal independent in the forest $R \backslash\{r\}$, the coefficient $b$ encodes maximal independent sets $\mathcal{I}$ of $R$ such that $r \notin \mathcal{I}$ and $c$ encodes maximal independent sets of $R$ containing the root vertex $r$ ).

For the tree underlying Figure 1 we get

$$
4 x^{3}+3 x^{4}+x^{5} .
$$

\subsubsection{Enumeration of (maximal) matchings}

A matching of a graph is a set of disjoint edges. The polynomial $\sum_{j} \alpha_{j} x^{j}$ with $\alpha_{j}$ counting matchings involving $j$ edges can be computed as the local invariant

$$
\begin{aligned}
v & =(1,0), \\
e(a, b) & =(a+b, x a), \\
m((a, b),(\alpha, \beta)) & =(a \alpha, a \beta+b \alpha), \\
f(a, b) & =a+b .
\end{aligned}
$$

We leave the easy details to the reader. (Hint: The first coefficient $a$ of $(a, b)$ counts matchings of a rooted tree not involving the root, the second coefficient $b$ counts matchings involving the root.) 
For the tree underlying Figure 1 we get

$$
1+7 x+13 x^{2}+7 x^{3} .
$$

A matching of a graph $G$ is maximal if it intersects every edge of $G$. The polynomial $\sum_{j} \beta_{j} x^{j}$ with $\beta_{j}$ counting maximal matchings involving $j$ edges can be computed as the local invariant

$$
\begin{aligned}
v & =(0,1,0) \\
e(a, b, c) & =(b, c, x(a+b)) \\
m((a, b, c),(\alpha, \beta, \gamma)) & =(a \alpha+a \beta+b \alpha, b \beta,(a+b) \gamma+c(\alpha+\beta)), \\
f(a, b, c) & =b+c
\end{aligned}
$$

( $a$ counts not maximal matchings of a rooted tree $R$ inducing maximal matchings on the forest $R \backslash\{r\}$ obtained by removing the root $r$ from $R$, the coefficient $b$ counts maximal matchings of $R$ not involving the root and $c$ counts maximal matchings of $R$ involving the root $r$ ).

For the tree underlying Figure 1 we get $7 x^{3}$.

\subsubsection{The characteristic polynomial of the adjacency matrix}

We write $(a, b) \in \mathbb{Z}[x]$ if the characteristic polynomial of a rooted tree is given by $a x-b$ with $a x$ corresponding to the contribution of the diagonal entry associated to the root. Elementary matrix-transformations show that the characteristic polynomial $\operatorname{det}(x \operatorname{Id}-A)$ of the adjacency matrix $A$ is a local invariant defined by

$$
\begin{aligned}
v & =(1,0), \\
e(a, b) & =(x a-b, a), \\
m((a, b),(\alpha, \beta)) & =(a \alpha, a \beta+b \alpha), \\
f(a, b) & =x a-b .
\end{aligned}
$$

For the tree underlying Figure 1 we get

$$
x^{8}-7 x^{6}+13 x^{4}-7 x^{2} .
$$

Similarly,

$$
\begin{aligned}
v & =(1,0,0), \\
e(a, b, c) & =(-(x+c+1) a+b,-a, 1), \\
m((a, b, c),(\alpha, \beta, \gamma)) & =(a \alpha, a \beta+b \alpha, c+\gamma), \\
f(a, b, c) & =-(x+c) a+b
\end{aligned}
$$

computes the characteristic polynomial of the combinatorial Laplacian (given by $D_{\operatorname{deg}}-A$ where $A$ is the adjacency matrix and $D_{\text {deg }}$ is the diagonal matrix defined by vertex-degrees) of a tree.

For the tree underlying Figure 1 we get

$$
x^{8}+14 x^{7}+76 x^{6}+204 x^{5}+286 x^{4}+204 x^{3}+67 x^{2}+8 x .
$$




\subsection{An example with values in $\mathbb{N}\left[z_{0}, z_{1}, \ldots\right]$}

Given a formal power series $Z=\sum_{i=0}^{\infty} z_{i} t^{i} \in \mathbb{A}[[t]]$ and a polynomial $B=\sum_{i=0}^{N} b_{i} t^{i} \in \mathbb{A}[t]$ with coefficients in a commutative ring $\mathbb{A}$, we denote by

$$
\langle Z, B\rangle=\sum_{i=0}^{N} z_{i} b_{i}
$$

the scalar product of the "coefficient-vectors" $\left(z_{0}, z_{1}, \ldots\right)$ and $\left(b_{0}, \ldots, b_{N}, 0, \ldots\right)$.

Proposition 5. We denote by $Z=\sum_{i=0}^{\infty} z_{i} t^{i}$ a formal power series in $t$ with coefficients $z_{i}$. The formulae

$$
\begin{aligned}
v & =1, \\
e(A) & =\langle Z, A\rangle+t A, \\
m(A, B) & =A B, \\
f(A) & =\langle Z, A\rangle
\end{aligned}
$$

define a local invariant of trees with values in $\mathbb{N}\left[z_{0}, z_{1}, z_{2}, \ldots\right]$, respectively of rooted trees with values in $\mathbb{N}\left[z_{0}, z_{1}, z_{2}, \ldots\right][t]$.

For the tree underlying Figure 1 we get

$$
\begin{aligned}
& z_{0}^{8}+7 z_{0}^{6} z_{1}+8 z_{0}^{5} z_{2}+z_{0}^{4}\left(13 z_{1}^{2}+10 z_{3}\right)+z_{0}^{3}\left(18 z_{1} z_{2}+11 z_{4}\right) \\
& +z_{0}^{2}\left(7 z_{1}^{3}+12 z_{1} z_{3}+3 z_{2}^{2}+8 z_{5}\right)+z_{0}\left(9 z_{1}^{2} z_{2}+8 z_{1} z_{4}+2 z_{2} z_{3}+4 z_{6}\right) \\
& +z_{1}^{2} z_{3}+2 z_{1} z_{2}^{2}+2 z_{1} z_{5}+z_{2} z_{4}+z_{7} .
\end{aligned}
$$

The specialization $z_{2}=z_{3}=\cdots=0$ of Proposition 5 is particularly interesting in the sense that it gives an invariant in $\mathbb{N}\left[z_{0}, z_{1}\right]([t])$ of (rooted) trees which behaves naturally with respect to the differential structure of $\mathbb{N}[\mathcal{R}]$. Given a rooted tree $R$ with invariant $c_{R}(0)+$ $c_{R}(1) t+\ldots$, the constant coefficient $c_{R}(0)$ corresponds to $R$ and the linear coefficient $c_{R}(1)$ corresponds to the derivative (as in Section 2.1) of $R$. The result (with $Z=z_{0}+z_{1} t$ ) for the tree underlying Figure 1 is

$$
z_{0}^{2}\left(z_{0}^{2}+z_{1}\right)\left(z_{0}^{4}+6 z_{0}^{2} z_{1}+7 z_{1}^{2}\right) .
$$

Proof of Proposition 5. The set $\mathbb{N}\left[z_{0}, z_{1}, z_{2}, \ldots\right][t]$ of all polynomials is a multiplicative monoid with product $m(A, B)=A B$ and identity 1 . The operator $e$ defines a map from $\mathbb{N}\left[z_{0}, z_{1}, z_{2}, \ldots\right][t]$ into itself. This shows that the formulae of Proposition 5 define a local invariant of rooted trees.

Symmetry in $A, B$ of

$$
\begin{aligned}
f(m(A, e(B))) & =f(A(\langle Z, B\rangle+t B)) \\
& =\langle Z, A\rangle\langle Z, B\rangle+\langle Z, t A B\rangle
\end{aligned}
$$

implies that identity (5) holds. 


\subsubsection{Examples}

Up to a power of $x$, the example of Section 2.4.2 enumerating matchings corresponds to $Z=\frac{1+t}{x}$ with coefficients $z_{0}=z_{1}=1 / x$ and $z_{2}=z_{3}=\cdots=0$.

Our next example, stated as a theorem, will be the crucial ingredient for proving Theorem 1:

Theorem 6. The characteristic polynomial of Section 2.4.3 corresponds to $Z=x-t$ with coefficients $z_{0}=x, z_{1}=-1$ and $z_{2}=z_{3}=\cdots=0$.

Proof. Follows easily from the formulae given in Section 2.4.3.

\section{A local invariant enumerating Schrödinger operators}

We consider the local invariant $S: \mathcal{T} \longrightarrow \mathbb{Z}[q]$ of trees given by the specialization $Z=$ $q-1+q t$ with coefficients $z_{0}=q-1, z_{1}=q$ and $z_{2}=z_{3}=\cdots=0$ of the local invariant described by Proposition 5 .

Since $S$ depends only on the constant and on the linear coefficient of the corresponding local invariant $a+b t+\ldots$ of rooted trees, we can also define $S$ by the formulae

$$
\begin{aligned}
v & =(1,0), \\
e(a, b) & =((q-1) a+q b, a), \\
m((a, b),(\alpha, \beta)) & =(a \alpha, a \beta+b \alpha), \\
f(a, b) & =(q-1) a+q b
\end{aligned}
$$

with $(a, b)$ representing the series expansion $a+b t+O\left(t^{2}\right)$.

For the (unrooted) tree underlying Figure 1 we get the polynomial

$$
(q-1)^{2}\left(q^{2}-q+1\right)\left(q^{4}+2 q^{3}+q^{2}+2 q+1\right) .
$$

Remark 7. As a mnemotechnical device, the formula for $m$ corresponds also to the addition $\frac{b}{a}+\frac{\beta}{\alpha}=\frac{a \beta+b \alpha}{a \alpha}$ with forbidden simplification and the formula for $e$ is, up to simplification by $a$, given by the homography $\frac{b}{a} \longmapsto\left(\begin{array}{cc}0 & 1 \\ q & q-1\end{array}\right) \frac{b}{a}=\frac{1}{q \frac{b}{a}+q-1}$. The formula for $f$ can be recovered from $e$ using the identity $e(a, b)=(f(a, b), a)$.

We write $S_{T}$ for the local invariant in $\mathbb{Z}[q]$ associated to a tree $T$. Similarly, given a rooted tree $R$, we denote by $S_{R}=(a, b) \in(\mathbb{Z}[q])^{2}$ the corresponding pair of polynomials defined by formulae (6).

Proposition 8. $S_{T}$ counts the number of invertible Schrödinger operators for a finite tree $T$ over the finite field $\mathbb{F}_{q}$.

A matrix $M$ with rows and columns indexed by vertices of a graph $G$ is a $G$-matrix if non-zero off-diagonal coefficients $m_{s, t}$ of $M$ correspond to edges $\{s, t\}$ of $G$. Diagonal entries of $G$-matrices are arbitrary. The off-diagonal support (set of non-zero coefficients) of a $G$-matrix encodes thus the edge-set of $G$. A $G$-matrix of an unoriented graph $G$ has always a symmetric support but is not necessarily symmetric. We have: 
Proposition 9. The number of invertible $T$-matrices over $\mathbb{F}_{q}$ of a finite tree $T$ having $n$ vertices is given by $(q-1)^{2 n-2} S_{T}$.

Proof of Proposition 8. We consider the obvious action on $T$-matrices of the abelian group $\left(\mathbb{F}_{q}^{*}\right)^{n} \times\left(\mathbb{F}_{q}^{*}\right)^{n}$ of pairs of invertible diagonal matrices (with coefficients in $\mathbb{F}_{q}$ ) by left and right multiplication. An orbit of a $T$-matrix without non-zero diagonal coefficients contains exactly one Schrödinger operator stabilized by a subgroup of order $(q-1)^{2}$. All other orbits contain exactly $q-1$ different Schrödinger operators, each stabilized by a subgroup of order $q-1$. The number of (invertible) $T$-matrices is thus exactly $(q-1)^{2 n-2}$ times larger than the number of (invertible) Schrödinger operators. The result follows now from Proposition 9.

Proof of Proposition 9. An $R$-matrix for a rooted tree $R \in \mathcal{R}$ is a $T$-matrix for the underlying unrooted tree $T$ with an unknown $x$ on the diagonal corresponding to the root of $R$. The determinant of an $R$-matrix over a finite field $\mathbb{F}_{q}$ is an affine function of the form $a x+b \in \mathbb{F}_{q}[x]$.

We consider now a fixed rooted tree $R$. Given two subsets $\mathcal{A}, \mathcal{B}$ of $\mathbb{F}_{q}$, we denote by $\nu(\mathcal{A}, \mathcal{B})$ the number of $R$-matrices of determinant $a x+b$ with $(a, b) \in \mathcal{A} \times \mathcal{B}$. We encode the natural integers $\nu(0,0), \nu\left(0, \mathbb{F}_{q}^{*}\right), \nu\left(\mathbb{F}_{q}^{*}, 0\right), \nu\left(\mathbb{F}_{q}^{*}, \mathbb{F}_{q}^{*}\right)$ (with 0 denoting the singleton subset $\{0\}$ consisting of the zero-element in $\mathbb{F}_{q}$ ) using the square matrix

$$
\left(\begin{array}{cc}
\nu(0,0) & \nu\left(0, \mathbb{F}_{q}^{*}\right) \\
\nu\left(\mathbb{F}_{q}^{*}, 0\right) & \nu\left(\mathbb{F}_{q}^{*}, \mathbb{F}_{q}^{*}\right)
\end{array}\right)
$$

Right and left multiplications by invertible diagonal matrices essentially preserve the set of $R$-matrices. More precisely, this holds up to replacement of the unknown $x$ by a nonzero multiple $\lambda x$ (with $\lambda \in \mathbb{F}_{q}^{*}$ ) of it. Since $x$ can be thought of as a simple place-holder for an arbitrary element of $\mathbb{F}_{q}$, such a scalar $\lambda$ can be dismissed. It follows that we have $\nu(\lambda a, \mu b)=\nu(a, b)$ (using a slight abuse of notation) if $\lambda$ and $\mu$ belong both to the set $\mathbb{F}_{q}^{*}$ of invertible elements in $\mathbb{F}_{q}$. Elementary linear algebra shows now that the operators $V, E, M, F$ correspond to the operators

$$
\begin{aligned}
v & =\left(\begin{array}{ll}
0 & 0 \\
1 & 0
\end{array}\right) \\
e & =\left(\begin{array}{ll}
a & b \\
c & d
\end{array}\right) \longmapsto(q-1)^{2}\left(\begin{array}{cc}
q a & c+d \\
q b & (q-1)(c+d)
\end{array}\right), \\
m & =\left(\left(\begin{array}{ll}
a & b \\
c & d
\end{array}\right),\left(\begin{array}{ll}
\alpha & \beta \\
\gamma & \delta
\end{array}\right)\right) \longmapsto\left(\begin{array}{cc}
A & B \\
C & D
\end{array}\right), \\
f & =\left(\begin{array}{ll}
a & b \\
c & d
\end{array}\right) \longmapsto q b+(q-1)(c+d)
\end{aligned}
$$

where

$$
A=a \alpha+a \beta+b \alpha+a \gamma+c \alpha+a \delta+d \alpha+b \beta
$$




$$
\begin{aligned}
B & =b \gamma+c \beta+b \delta+d \beta \\
C & =c \gamma+\frac{1}{q-1} d \delta \\
D & =c \delta+d \gamma+\frac{q-2}{q-1} d \delta
\end{aligned}
$$

The coefficient $\nu(0,0)$ contributes never to the number of invertible Schrödinger operators and can be discarded. Inspection of the above formulae shows that we can lump together $\nu\left(\mathbb{F}_{q}^{*}, 0\right)$ and $\nu\left(\mathbb{F}_{q}^{*}, \mathbb{F}_{q}^{*}\right)$ into a first coordinate with the second coordinate given by $\nu\left(0, \mathbb{F}_{q}^{*}\right)$. This leads to the formulae (6) for $S_{T}$, except for an extra factor of $(q-1)^{2}$ for every edge of $T$.

\subsection{Proof of Theorem 1}

Substituting $x$ with $\sqrt{-q}+1 / \sqrt{-q}$ and multiplying by the correct sign and power of $\sqrt{-q}$, the expression $(-\sqrt{-q})^{n} \chi_{T}(\sqrt{-q}+1 / \sqrt{-q})$ (with $n$ denoting the number of vertices of $T$ ) can be computed using Theorem 6 as the local invariant given by

$$
\begin{aligned}
v & =1, \\
e\left(a+t b+O\left(t^{2}\right)\right) & =-\sqrt{-q}(\sqrt{-q}+1 / \sqrt{-q}) a-\sqrt{-q}^{2} b+a t+O\left(t^{2}\right) \\
& =(q-1) a+q b+a t+O\left(t^{2}\right), \\
m(A, B) & =A B \\
f\left(a+t b+O\left(t^{2}\right)\right) & =-\sqrt{-q}(\sqrt{-q}+1 / \sqrt{-q}) a-\sqrt{-q}^{2} b \\
& =(q-1) a+q b
\end{aligned}
$$

and coincides thus with the local invariant given by formulae (6) which define the counting function $S_{T}$ for Schrödinger operators by Proposition 8 .

\section{A few additional properties of $S_{T}$}

Since Theorem 1 links $S_{T}$ closely to the characteristic polynomial (of an adjacency matrix), many properties of $S_{T}$ mirror properties of characteristic polynomials for trees. For example, since characteristic polynomials (of integral matrices) are monic and integral, the polynomial $S_{T}$ is monic and integral.

A property not linked to the characteristic polynomial but to the formula (1) defining $S_{T}$ is the fact that the polynomial $S_{T}$ associated to a tree $T$ with $n$ vertices satisfies the equation

$$
S_{T}(q)=(-q)^{n} S_{T}(1 / q) .
$$

We call this property sign-degree-palindromicity. It implies that a complex number $\rho$ is a root of $S_{T}$ if and only if $1 / \rho$ is a root.

Another easy fact (left to the reader), is the observation that $S_{T}$ is always of the form $q^{n}-q^{n-1}+\ldots$ This is (up to $O\left(q^{n-2}\right)$ ) the expected number of non-zero elements among $q^{n}$ (uniformly distributed) random elements of $\mathbb{F}_{q}$. 


\subsection{Root-locus of $S_{T}$}

All roots of the characteristic polynomial of a graph are real. Since a tree $T$ is bipartite, a real number $\rho$ is a root of $\chi$ (the characteristic polynomial of an adjacency matrix of $T$ ) if and only if $-\rho$ is a root. A pair of non-zero roots $\pm \rho$ of $\chi$ gives thus rise to the pair $\sigma, 1 / \sigma$ of roots of $S_{T}$ satisfying the equation

$$
\begin{aligned}
0 & =(\sqrt{-\sigma}+1 / \sqrt{-\sigma}-\rho)(\sqrt{-\sigma}+1 / \sqrt{-\sigma}+\rho) \\
& =-\sigma+2-1 / \sigma-\rho^{2} .
\end{aligned}
$$

We have thus

$$
\sigma^{ \pm 1}=\frac{2-\rho^{2} \pm \sqrt{\left(\rho^{2}-2\right)^{2}-4}}{2} .
$$

For $\rho \in[-2,2]$ we get two conjugate roots $\sigma, \bar{\sigma}=1 / \sigma$ on the complex unit circle (except perhaps for $\rho=0$ giving sometimes rise to a unique root 1 of $S_{T}$ ), for $\rho$ of absolute value larger than 2 we get two negative real roots $\sigma, 1 / \sigma$.

All roots of $S_{T}$ are thus on the union of the complex unit circle with the real negative half-line.

Trees with all roots of $S_{T}$ on the unit circle are subtrees of affine Dynkin diagrams of type $D$ or $E$, see for example [2] which describes also all trees such that $S_{T}$ has exactly one real root $<-1$. (More precisely, [2] deals with polynomials of the form (1) (up to trivial signs) which give rise to Salem numbers.)

Our next result shows that "simple" trees give rise to polynomials $S_{T}$ with few real negative roots:

Proposition 10. The number of real negative zeroes of $S_{T} \in \mathbb{Z}[q]$ associated to a tree $T$ is at most equal to twice the number of non-root vertices of degree at least 3 in $T$.

The proof uses the following auxiliary result:

Lemma 11. The numbers of real negative zeroes of the polynomials $a, b$ associated by $S_{R}=(a, b)$ to a rooted tree $R$ are at most equal to twice the number of non-root vertices of degree at least 3 in $R$.

Proof of Proposition 10. We turn $T$ into a rooted tree $R$ by choosing a root vertex at a leaf of $T$. The result follows now by applying Lemma 11 to the first polynomial of $S_{E(R)}=\left(S_{T}, *\right)$.

Proof of Lemma 11. Let $R$ be a rooted tree. If the root vertex $v_{*}$ of $R$ is not a leaf, then $S(R)=(A, B)=(a \alpha, a \beta+b \alpha)$ where $(a, b)$ and $(\alpha, \beta)$ are associated to smaller nontrivial rooted trees $R_{1}, R_{2}$ such that $R=M\left(R_{1}, R_{2}\right)$. The result holds thus for $A=a \alpha$ by induction on the number of vertices and it holds for $B$ since roots of $A$ and $B$ interlace in an obvious sense on $\mathbb{S}^{1} \cup \mathbb{R}_{<0}$. If the root vertex $v_{*}$ is a leaf, the result holds by a straightforward computation if $R$ is a leaf-rooted path (Dynkin diagram of type $A$ ). Otherwise, the tree $R$ contains a vertex $w$ of degree at least 3. Working with the rooted tree $R_{w}$ corresponding to $T$ rooted at $w$, we see that $a_{w}, b_{w}$ with $S\left(R_{w}\right)=\left(a_{w}, b_{w}\right)$ have 
at most $2(k-1)$ real negative zeroes where $k$ is the number of vertices of degree at least 3 in $T$. This implies that $S_{T}$ has at most $2+2(k-1)=2 k$ real negative zeroes. Since $S_{T}$ has at least as many real negative zeroes as $b$ involved in $S(R)=(a, b)$ and since roots of $a$ and $b$ interlace, the polynomials $a, b$ have both at most $2 k$ real zeroes.

\subsection{Multiple roots of $S_{T}$}

A vertex $w$ of degree $k$ of a tree $T$ can be considered as the result of gluing $k$ maximal leaf-rooted subtrees of $T$ along their root-leaf corresponding to $w$. This construction is linked to some multiple roots of $S_{T}$ as follows: Given a leaf-rooted tree $R$, let $w_{1}, \ldots, w_{k}$ be the list of all vertices of $T$ involving $R$ (i.e. at least one of the maximal leaf-rooted subtrees of $T$ with root $w_{i}$ is isomorphic to $R$ ). Given such a vertex $w_{i}$, we denote by $r_{i}+1 \geqslant 1$ the number of occurrences of $R$ at $w_{i}$. We have the following result:

Proposition 12. The polynomial $S_{T}$ is (at least) divisible by $a^{r}$ where $r=r_{1}+\cdots+r_{k}$ and where $a$ is defined by $S_{R}=(a, b)$.

Proof. Gluing $k_{i}+1$ copies of $R$ along their root gives a rooted tree with invariant $\left(a^{k_{i}+1},\left(k_{i}+1\right) a^{k_{i}} b\right)$. Linearity of the formulae for $e, f$ and $m$ implies now the result.

Proposition 12 explains the factor $q^{2}-q+1$ and one of the factors $q-1$ of the polynomial $S_{T}=(q-1)^{2}\left(q^{2}-q+1\right)\left(q^{4}+2 q^{3}+q^{2}+2 q+1\right)$ with $T$ given by Figure 1 . Since $S_{T}$ is palindromic, the factor $q-1$ divides $S_{T}$ with even multiplicity. All cyclotomic factors in this example have thus easy explanations.

\section{$5 \quad$ Trees with coloured vertices}

A rooted tree with ordinary (non-root) vertices coloured (not necessarily properly, i.e. adjacent vertices might have identical colours) by a set $\mathcal{C}$ can be constructed using the construction-operators $V$ (creation of a root-vertex), $M$ (merging of two rooted trees along their root) and replacing $E$ by operators $E_{c}$ (for $c \in \mathcal{C}$ ) depending on the final colour of the initial root-vertex. For ordinary trees, one works with operators $F_{c}$ indexed by all possibilities of colouring the root-vertex after turning it into an ordinary vertex.

Identity (5) has to be replaced by

$$
F_{s}\left(M\left(A, E_{t}(B)\right)=F_{t}\left(M\left(E_{s}(A), B\right)\right)\right.
$$

for all $s, t \in \mathcal{C}$ and for all $A, B \in \mathcal{R}$.

Local invariants for coloured (rooted or plane) trees are defined in the obvious way.

\subsection{A few examples of coloured invariants}

\subsubsection{Colourings defined by (virtual) rooted trees}

Every local invariant gives rise to a coloured local invariant by chosing colour-constants $u_{c} \in \mathbb{E}$ for all colours $c \in \mathcal{C}$ and by replacing $e$ with $e_{c}(A)=e\left(m\left(A, u_{c}\right)\right)$ and $f$ with 
$f_{c}(A)=f\left(m\left(A, u_{c}\right)\right)$. These invariants amount to attaching "virtual trees" $U_{c}$ corresponding to $u_{c}$ and representing colours to all ordinary vertices.

A particular case, closely related to $S_{T}$, will be discussed in Section 6.2.

\subsubsection{A coloured local invariant with values in $\mathbb{N}\left[z_{0}(c), z_{1}, z_{2}, z_{3}, \ldots\right]$}

The example of Proposition 5 in Section 2.5 can easily be generalized to a coloured local invariant by considering formal power-series $Z(s)=z_{0}(s)+\sum_{i=1}^{\infty} z_{i} t^{i}$ with constant terms (with respect to $t$ ) depending on colours. Identity (8), corresponding to

$$
\left\langle Z\left(s_{1}\right), A\right\rangle\left\langle Z\left(s_{2}\right), B\right\rangle+\left\langle Z\left(s_{1}\right), t A B\right\rangle=\left\langle Z\left(s_{1}\right), A\right\rangle\left\langle Z\left(s_{2}\right), B\right\rangle+\left\langle Z\left(s_{2}\right), t A B\right\rangle,
$$

holds since $\langle Z(s), t C\rangle$ does not depend on the colour $s$.

As an example, we can consider the invariant given by $Z(v)=x_{v}+q t$ generalizing the invariant counting invertible Schrödinger obtained by the specialization $x_{v}=q-1$ for all $v$, see Section 3.

\subsubsection{Bicoloured characteristic polynomial}

Trees are connected bipartite graphs and have thus a canonical proper 2-colouring or bicolouring, well-defined up to colour-exchange. The corresponding bi-coloured variation of the characteristic polynomial of the adjacency matrix is given by computing the determinant of the matrix coinciding with the adjacency matrix outside the diagonal and with diagonal coefficients $-x$ or $-y$ according to the bipartite class of the corresponding vertex. The resulting determinant is well-defined in $\mathbb{Z}[x, y]$ up to exchanging $x$ with $y$ and can be computed as a local invariant. This construction works of course also for the combinatorial Laplacian of a tree.

\subsubsection{Coloured Schrödinger operators}

The enumeration of Schrödinger operators according to coloured diagonal zeros leads to a local invariant of coloured trees. It takes its values in $\mathbb{Z}[q, \mathcal{C}]$ with the coefficient (in $\mathbb{Z}[q])$ of a monomial $\prod_{j} c_{j}^{e_{j}} \in \mathcal{C}^{*}$ counting the number of Schrödinger operators with $e_{j}$ zero terms on diagonal elements associated to vertices of colour $c_{j}$.

The corresponding operators are given by

$$
\begin{array}{ll}
v & =(0,1,0), \\
e_{s}(a, b, c) & =(s b+c,(q-1+s) a,(q-1) b+(q-2+s) c), \\
m((a, b, c),(\alpha, \beta, \gamma)) & =\left(a \beta+b \alpha+a \gamma+c \alpha, b \beta+\frac{c \gamma}{q-1}, b \gamma+c \beta+\frac{q-2}{q-1} c \gamma\right) \\
f_{s}(a, b, c) & =(q-1+s) a+(q-1) b+(q-2+s) c .
\end{array}
$$

(with $(a, b, c)$ standing for $a=N\left(0, \mathbb{F}_{q}^{*}\right), b=N\left(\mathbb{F}_{q}^{*}, 0\right)$ and $c=N\left(\mathbb{F}_{q}^{*}, \mathbb{F}_{q}^{*}\right)$ where $N(*, *)$ is as in the proof of Proposition 9).

Skeptical readers are invited to check the identity

$$
f_{s}\left(m\left((a, b, c), e_{t}(\alpha, \beta, \gamma)\right)\right)=f_{t}\left(m\left(e_{s}(a, b, c),(\alpha, \beta, \gamma)\right)\right)
$$

corresponding to (8). 


\subsection{A further generalization: working with coloured monoids}

Rooted vertex-coloured trees with colours at all vertices including the root do not form a natural monoid. However the subset of rooted trees with a given root colour is obviously a monoid (by gluing, as before, all root-vertices into a root-vertex of the same colour).

A local invariant for such rooted coloured trees is given by monoids $\mathcal{M}_{c}$ with identities $v_{c}$ and products $m_{c}$ and by edge-maps $e_{c_{i}, c_{f}}: \mathcal{M}_{c_{i}} \longrightarrow \mathcal{M}_{c_{f}}$ (depending on the root-colour $c_{i}$ of the initial argument-tree and on the colour $c_{f}$ of the final, added root-vertex) among all coloured monoids.

Adding maps $f_{c}: \mathcal{M}_{c} \longrightarrow \mathbb{F}$ into some set $\mathbb{F}$ such that we have for all pairs of colours $c_{1}, c_{2}$ the identity

$$
f_{c_{1}}\left(m_{c_{1}}\left(A_{1}, e_{c_{2}, c_{1}}\left(A_{2}\right)\right)\right)=f_{c_{2}}\left(m_{c_{2}}\left(e_{c_{1}, c_{2}}\left(A_{1}\right), A_{2}\right)\right)
$$

(with $A_{1} \in \mathcal{M}_{c_{1}}, A_{2} \in \mathcal{M}_{c_{2}}$ ), we get an invariant of coloured trees.

Section 7 contains an example of this construction.

\section{Edge-subdivisions}

\subsection{Properties of $S$}

Proposition 13. Let $T_{i}$ be a sequence of trees obtained by subdividing all edges around a fixed vertex $w$ of degree $d \geqslant 3$ of a finite tree $T$ into a larger and larger number of edges (by insertion of additional vertices of degree 2). Then there exists a sequence of roots $\rho_{i}$ of $S_{T_{i}}$ converging to $1-d$.

The proof follows easily from the discussions in Section 6.2.

Applying Proposition 13 at all vertices we get:

Corollary 14. Subdividing all edges of a fixed finite tree $T$ leads to a sequence of polynomials with $k$ strictly negative roots converging to $1-d_{i}$ where $d_{1}, \ldots, d_{k}$ are the degrees of all $k$ vertices of $T$ with degrees $>2$.

The density of roots on $\mathbb{S}^{1}$ under edge-subdivisions can be shown to behave as expected:

Proposition 15. Given a sequence $T_{i}$ of finite trees obtained by subdividing some edges of a given fixed tree $T$ into more and more sub-edges, the density of roots of $S_{T_{i}}$ on the unit circle $\mathbb{S}^{1}$ converges to the Lebesgue measure of $\mathbb{S}^{1}$. Otherwise stated, the proportion of roots of $S_{T_{i}}$ in a given sub-interval I of $\mathbb{S}^{1}$ tends to $\frac{1}{2 \pi}$ length $(I)$.

We omit the proof.

\subsection{Essentially finite trees and limits of real negative roots of $S_{T}$ under edge- subdivisions}

A perhaps infinite tree is essentially finite if it is obtained by subdiving edges of a finite tree at most countably many times. Essentially finite trees can be considered as trees with 
edges weighted by elements of $\{1,2, \ldots\} \cup\{\infty\}$. Edge-weights encode the final number of edges after subdivision. Forbidding vertices of degree 2 leads to unique representations of this form.

Essentially finite trees with an infinite number of vertices have no longer an $S$ polynomial. They define however a finite set $\rho_{1}, \ldots, \rho_{k}$ of $k$ real numbers $<-1$ where $k$ is at most equal to the number of vertices of degree $\geqslant 3$ in the following way: Approximate such a tree $T$ by a sequence $T_{i}$ of finite trees in the obvious way (by replacing all infinite edge-weights by large finite edge-weights) and consider the limits (which exist by Theorem 16) of all real roots $<-1$ of the polynomials $S_{T_{i}}$, taking into account multiplicities. The aim of this section is to compute these numbers and to study a few of their properties.

An essentially finite tree is arc-connected if all non-leaves are at finite distance. Equivalently, a finite tree is arc-connected if all its edges with infinite weight contain a leaf. An arc-connected component of an essentially finite tree is a subtree defined by all vertices at finite distance of a non-leaf. An arc-connected essentially finite tree is a finite tree together with attachements of finitely many infinite rays (ending at an "ideal" leaf-vertex) at vertices. An essentially finite arc-connected tree can be encoded by a finite tree with $\mathbb{N}$-weighted vertices. Vertex-weights indicate numbers of attached infinite rays.

Arbitrary essentially finite trees can be decomposed into essentially finite arc-connected trees with two arc-connected components intersecting at most in a unique "ideal midpoint" of an infinitely subdivised edge.

The data

$$
\begin{array}{ll}
v & =(1,0) \\
e_{k}(a, b) & =(q(-k a+b)+(q-1) a, a), \\
m((a, b),(\alpha, \beta)) & =(a \alpha, a \beta+b \alpha) \\
f_{k}(a, b) & =q(-k a+b)+(q-1) a
\end{array}
$$

defines a local invariant for coloured trees with colour-values in some ring. We leave it to the reader to check that (8) holds. This local invariant corresponds to a coloured invariant where a vertex $v$ of weight $k$ is decorated by a non-existent ideal rooted tree with $S$-polynomial $(1,-k)$, see also Section 5.1.1. It can also be considered as the specialization given by $Z(k)=(q-1-q k)+q t$ of the invariant described in Section 5.1.2.

We denote by $Q_{T} \in \mathbb{Z}[q]$ the polynomial defined by (10) for a finite tree $T$ with $\mathbb{Z}$-coloured vertices.

For values in $\mathbb{N}$ encoding numbers of "limit rays" of an arc- connected essentially finite tree, the polynomial $Q_{T}$ has the inverse limit values $1 / \rho_{i} \in(-1,0)$ (taking multiplicities into account) among its roots. In particular, the limit-values $\rho_{i}$ are algebraic numbers (in fact algebraic integers since $Q_{T}(0) \in\{ \pm 1\}$ ) of degrees bounded by the number of vertices in a $\mathbb{N}$-vertex-coloured finite tree representation. Indeed, given a sequence $R_{i}$ of increasing leaf-rooted paths (Dynkin diagrams of type $A$ ), the evaluation of $S_{R_{i}}$ at a complex number $\rho$ of norm $<1$ tends to $\pm \frac{1}{1+\rho}(1,-1)$ (with a sign depending on the parity of the number of vertices). Linearity of the maps $e, m$ and $f$ implies now that the limit values $\rho_{i}$ are algebraic numbers.

Given a sequence $T_{i}$ of finite trees obtained by subdividing increasingly often a unique 
edge of an (essentially finite) tree, the argument above shows that a real limit-root $\rho$ "belongs" to one of the two arc-connected components of the essentially finite limit-tree.

Proof of Proposition 13. Follows from the fact that the essentially finite arc-connected tree $T$ represented by an isolated vertex of weight $d \geqslant 3$ gives rise to $Q_{T}=f(1,-d)=$ $(1-d) q-1$.

We have:

Theorem 16. All non-limit roots of $Q_{T}$ have norm $\geqslant 1$ if $T$ encodes an essentially finite arc-connected tree (i.e. if all vertex-colours are in $\mathbb{N}$ ).

Proof. Let $\sigma$ be a root of $Q_{T}$ in the open complex unit disc. The root $\sigma$ can be approximated with arbitrary accuracy by a root of $S_{T^{\prime}}$ where $T^{\prime}$ is a finite tree approximating the essentially finite tree $T$. This implies that $\sigma$ has to be a real negative number, see Section 4.1 .

We call the sum of vertex-weights the weight-degree of a $\mathbb{N}$-coloured connected essential tree $T$.

Trees of degree 0 are ordinary trees. Their $S$-polynomials define in some sense "generalized Salem numbers". Trees of degree 1 define "generalized Pisot numbers" as accumulation points of "generalized Salem numbers". Trees of degree $\geqslant 2$ lead to iterated accumulation points.

\section{Counting Schrödinger operators according to values of the Jacobi symbol}

We describe a local invariant for computing the number of Schrödinger operators of a tree over a field $\mathbb{F}_{q}$ of odd characteristic according to values of the Jacobi-symbol (corresponding to coefficients which are zero, non-zero squares or non-squares) at diagonal entries indexed by vertices.

Our formulae define in fact a coloured local invariant as defined in Section 5.2 with five free parameters $\epsilon_{v}, q_{v}, s_{v}, x_{v}, y_{v}$ for each vertex $v$ and an additional global parameter $q$. The parameters $\epsilon_{v}, q_{v}$ (and the global parameter $q$ ) are involved in the monoid-product which depends thus on the root-colour, see Section 5.2.

Some specializations of this invariant count invertible Schrödinger operators with various restrictions (having for example only non-zero squares on the diagonal, or zeros, non-zero squares, respectively non-squares on selected subsets of diagonal entries).

Verifications are straightforward but tedious and are omitted.

We denote by $\mathcal{O}$ either the zero element of $\mathbb{F}_{q}$ or the element [0] of the group-algebra $\mathbb{Q}\left[\mathbb{F}_{q}\right]$ of the additive group $\mathbb{F}_{q}$. Similarly, $\mathcal{S}$ is either the set of all non-zero squares of the field $\mathbb{F}_{q}$ or the weighted sum $\frac{2}{q-1} \sum_{s \in \mathcal{S}}[s]$ of all non-zero squares in $\mathbb{Q}\left[\mathbb{F}_{q}\right]$, with equal weights summing up to 1 . We define $\mathcal{N}$ analogously using non-squares. 
Addition-rules for the elements $\mathcal{S}, \mathcal{N}$ (corresponding to the product in the groupalgebra $\mathbb{Q}\left[\mathbb{F}_{q}\right]$ of the additive group $\left.\left(\mathbb{F}_{q},+\right)\right)$ are given by

\begin{tabular}{c|cc} 
& $\mathcal{S}$ & $\mathcal{N}$ \\
\hline $\mathcal{S}$ & $\frac{1+\epsilon}{q-1} \mathcal{O}+\frac{q-4-\epsilon}{2(q-1)} \mathcal{S}+\frac{q-\epsilon}{2(q-1)} \mathcal{N}$ & $\frac{1-\epsilon}{q-1} \mathcal{O}+\frac{q-2+\epsilon}{2(q-1)}(\mathcal{S}+\mathcal{N})$ \\
$\mathcal{N}$ & $\frac{1-\epsilon}{q-1} \mathcal{O}+\frac{q-2+\epsilon}{2(q-1)}(\mathcal{S}+\mathcal{N})$ & $\frac{1+\epsilon}{q-1} \mathcal{O}+\frac{q-\epsilon}{2(q-1)} \mathcal{S}+\frac{q-4-\epsilon}{2(q-1)} \mathcal{N}$
\end{tabular}

where $\epsilon \in \pm 1$ satisfies $q \equiv \epsilon(\bmod 4)$.

Addition-rules in $\mathbb{Q}\left[\mathbb{F}_{q}\right]$ with $\mathcal{O}$ are of course given by $\mathcal{O}+X=X$ for $X \in\{\mathcal{O}, S, \mathcal{N}\}$.

Multiplication-rules are given by $\mathcal{O}^{2}=\mathcal{O}, \mathcal{O} \cdot \mathcal{S}=\mathcal{O} \cdot \mathcal{N}=\mathcal{O}, \mathcal{S}^{2}=\mathcal{N}^{2}=\mathcal{S}$ and $\mathcal{S N}=\mathcal{N}$. In particular, the element $\mathcal{S}$ is a multiplicative unity.

Given a rooted tree $R$ and two subsets $\mathcal{A}, \mathcal{B}$ of a finite field $\mathbb{F}_{q}$, we use the conventions of Section 3 and we denote by $\nu(\mathcal{A}, \mathcal{B})$ the number of Schrödinger operators with diagonals in $\mathbb{F}_{q}$ of the rooted tree $R$ having determinants of the form $\alpha x+\beta$ with $\alpha \in \mathcal{A}$ and $\beta \in \mathcal{B}$. We denote by $\mathcal{O}=\{0\}$ the zero element of $\mathbb{F}_{q}$ and by $\mathcal{S}$, respectively $\mathcal{N}$ the set of squares, respectively non-squares in $\mathbb{F}_{q}$ where we assume that $q$ is a power of an odd prime. We display all possible numbers $\nu(\mathcal{A}, \mathcal{B})$ with $\mathcal{A}, \mathcal{B} \in\{\mathcal{O}, \mathcal{S}, \mathcal{N}\}$ of a rooted tree $R$ as a square-matrix with rows and columns indexed by $\mathcal{O}, \mathcal{S}$ and $\mathcal{N}$ by writing

$$
\left(\begin{array}{ccc}
\nu(\mathcal{O}, \mathcal{O}) & \nu(\mathcal{O}, \mathcal{S}) & \nu(\mathcal{O}, \mathcal{N}) \\
\nu(\mathcal{S}, \mathcal{O}) & \nu(\mathcal{S}, \mathcal{S}) & \nu(\mathcal{S}, \mathcal{N}) \\
\nu(\mathcal{N}, \mathcal{O}) & \nu(\mathcal{N}, \mathcal{S}) & \nu(\mathcal{N}, \mathcal{N})
\end{array}\right)
$$

The number $\nu(\mathcal{O}, \mathcal{O})$ is in fact useless from our point of view but it allows for some numerical consistency checks.

The element

$$
v=\left(\begin{array}{lll}
0 & 0 & 0 \\
1 & 0 & 0 \\
0 & 0 & 0
\end{array}\right)
$$

is the identity of monoid structures with products given by

$$
m_{v}\left(\left(\begin{array}{lll}
a_{1} & b_{1} & c_{1} \\
d_{1} & e_{1} & f_{1} \\
g_{1} & h_{1} & i_{1}
\end{array}\right),\left(\begin{array}{ccc}
a_{2} & b_{2} & c_{2} \\
d_{2} & e_{2} & f_{2} \\
g_{2} & h_{2} & i_{2}
\end{array}\right)\right)=\left(\begin{array}{ccc}
A & B & C \\
D & E & F \\
G & H & I
\end{array}\right)
$$

where

$$
\begin{aligned}
A= & a_{1} a_{2}+a_{1}\left(b_{2}+c_{2}+d_{2}+e_{2}+f_{2}+g_{2}+h_{2}+i_{2}\right) \\
& +\left(b_{1}+c_{1}+d_{1}+e_{1}+f_{1}+g_{1}+h_{1}+i_{1}\right) a_{2}+\left(b_{1}+c_{1}\right)\left(b_{2}+c_{2}\right) \\
B= & b_{1}\left(d_{2}+e_{2}+f_{2}\right)+c_{1}\left(g_{2}+h_{2}+i_{2}\right) \\
& +\left(d_{1}+e_{1}+f_{1}\right) b_{2}+\left(g_{1}+h_{1}+i_{1}\right) c_{2} \\
C= & c_{1}\left(d_{2}+e_{2}+f_{2}\right)+b_{1}\left(g_{2}+h_{2}+i_{2}\right) \\
& +\left(d_{1}+e_{1}+f_{1}\right) c_{2}+\left(g_{1}+h_{1}+i_{1}\right) b_{2}
\end{aligned}
$$




$$
\begin{aligned}
D= & d_{1} d_{2}+g_{1} g_{2}+\frac{1+\epsilon_{v}}{q-1}\left(e_{1} e_{2}+i_{1} i_{2}+f_{1} f_{2}+h_{1} h_{2}\right) \\
& +\frac{1-\epsilon_{v}}{q-1}\left(e_{1} f_{2}+f_{1} e_{2}+h_{1} i_{2}+i_{1} h_{2}\right) \\
E= & d_{1} e_{2}+e_{1} d_{2}+g_{1} i_{2}+i_{1} g_{2}+\frac{q_{v}-4-\epsilon_{v}}{2(q-1)}\left(e_{1} e_{2}+i_{1} i_{2}\right) \\
& +\frac{\tilde{q}_{v}-2+\epsilon_{v}}{2(q-1)}\left(e_{1} f_{2}+f_{1} e_{2}+h_{1} i_{2}+i_{1} h_{2}\right)+\frac{q_{v}-\epsilon_{v}}{2(q-1)}\left(f_{1} f_{2}+h_{1} h_{2}\right) \\
F= & d_{1} f_{2}+f_{1} d_{2}+g_{1} h_{2}+h_{1} g_{2}+\frac{\tilde{q}_{v}-4-\epsilon_{v}}{2(q-1)}\left(f_{1} f_{2}+h_{1} h_{2}\right) \\
& +\frac{q_{v}-2+\epsilon_{v}}{2(q-1)}\left(e_{1} f_{2}+f_{1} e_{2}+h_{1} i_{2}+i_{1} h_{2}\right)+\frac{\tilde{q}_{v}-\epsilon_{v}}{2(q-1)}\left(e_{1} e_{2}+i_{1} i_{2}\right) \\
G= & d_{1} g_{2}+g_{1} d_{2}+\frac{1-\epsilon_{v}}{q-1}\left(e_{1} h_{2}+h_{1} e_{2}+f_{1} i_{2}+i_{1} f_{2}\right) \\
& +\frac{1+\epsilon_{v}}{q-1}\left(e_{1} i_{2}+i_{1} e_{2}+f_{1} h_{2}+h_{1} f_{2}\right) \\
H= & d_{1} h_{2}+h_{1} d_{2}+f_{1} g_{2}+g_{1} f_{2}+\frac{\tilde{q}_{v}-4-\epsilon_{v}}{2(q-1)}\left(f_{1} h_{2}+h_{1} f_{2}\right) \\
& +\frac{q_{v}-2+\epsilon_{v}}{2(q-1)}\left(e_{1} h_{2}+h_{1} e_{2}+f_{1} i_{2}+i_{1} f_{2}\right)+\frac{\tilde{q}_{v}-\epsilon_{v}}{2(q-1)}\left(e_{1} i_{2}+i_{1} e_{2}\right) \\
I= & d_{1} i_{2}+i_{1} d_{2}+e_{1} g_{2}+g_{1} e_{2}+\frac{q_{v}-4-\epsilon_{v}}{2(q-1)}\left(e_{1} i_{2}+i_{1} e_{2}\right) \\
& +\frac{\tilde{q}_{v}-2+\epsilon_{v}}{2(q-1)}\left(e_{1} h_{2}+h_{1} e_{2}+f_{1} i_{2}+i_{1} f_{2}\right)+\frac{q_{v}-\epsilon_{v}}{2(q-1)}\left(f_{1} h_{2}+h_{1} f_{2}\right) \\
H &
\end{aligned}
$$

and where $q_{v}, \tilde{q}_{v}$ satisfy

$$
q_{v}+\tilde{q}_{v}=2 q
$$

and have thus only one degree of freedom at a given vertex since $q$ is global (i.e. independent of vertices).

$\epsilon_{v}$ are also local variables.

It can be checked that $m_{v}$ defines for all $q, q_{v}, \epsilon_{v}$ (with $\tilde{q}_{v}=2 q-q_{v}$ ) a commutative and associative product with identity $v$.

The reader should be aware that we use the same letter $v$ for a vertex, its colour and the identity of colour $v$ with respect to the monoid with product $m_{v}$.

Edge-operators do not depend on the final root-colour which is thus omitted. The index $v$ in our formulae denotes the root-vertex (or its colour) of the rooted tree given as the argument. The edge-operator $e_{v}$ is given by

$$
e_{v}\left(\left(\begin{array}{ccc}
a & b & c \\
d & e & f \\
g & h & i
\end{array}\right)\right)=\left(\begin{array}{ccc}
A & \frac{1+\epsilon_{v}}{2} B_{+}+\frac{1-\epsilon_{v}}{2} B_{-} & \frac{1+\epsilon_{v}}{2} C_{+}+\frac{1-\epsilon_{v}}{2} C_{-} \\
D & \frac{1+\epsilon_{v}}{2} E_{+}+\frac{1-\epsilon_{v}}{2} E_{-} & \frac{1+\epsilon_{v}}{2} F_{+}+\frac{1-\epsilon_{v}}{2} F_{-} \\
G & \frac{1+\epsilon_{v}}{2} H_{+}+\frac{1-\epsilon_{v}}{2} H_{-} & \frac{1+\epsilon_{v}}{2} I_{+}+\frac{1-\epsilon_{v}}{2} I_{-}
\end{array}\right)
$$


(using the conventions of Section 3, except for a factor $((q-1) / 2)^{2}$ corresponding to arbitrary non-zero square values on oriented edges, when working with the specialization $q_{v}=q$ an odd prime power and $\epsilon_{v} \in\{ \pm 1\}$ given by $\left.q \equiv \epsilon_{v}(\bmod 4)\right)$ where

$$
\begin{gathered}
A=\left(s_{v}+\frac{q_{*}-1}{2}\left(x_{v}+y_{v}\right)\right) a \\
B_{+}=s_{v} d+x_{v} e+y_{v} f \\
B_{-}=s_{v} g+x_{v} h+y_{v} i \\
C_{+}=s_{v} g+x_{v} i+y_{v} h \\
C_{-}=s_{v} d+x_{v} f+y_{v} e \\
E_{+}=\left(s_{v}+\frac{q-1}{2}\left(x_{v}+y_{v}\right)\right) b \\
E_{-}=s_{v} h+\frac{q-1}{2} x_{v} d+\frac{q-1}{2} y_{v} g+\frac{q_{v}-4-\epsilon_{v}}{4} x_{v} e+\frac{\tilde{q}_{v}-2+\epsilon_{v}}{4}\left(x_{v} f+\epsilon_{v} e\right)+\frac{q_{v}-\epsilon_{v}}{4} y_{v} f+\frac{q_{v}-2+\epsilon_{v}}{4}\left(x_{v} h+y_{v} i\right)+\frac{\tilde{q}_{v}-\epsilon_{v}}{4} x_{v} i \\
F_{+}=s_{v} h+\frac{q-1}{2} y_{v} g+\frac{\tilde{q}_{v}-4-\epsilon_{v}}{4} y_{v} h+\frac{q_{v}-2+\epsilon_{v}}{4}\left(x_{v} h+y_{v} i\right)+\frac{\tilde{q}_{v}-\epsilon_{v}}{4} x_{v} i \\
F_{-}=s_{v} e+\frac{q-1}{2} x_{v} d+\frac{q_{v}-4-\epsilon_{v}}{4} x_{v} e+\frac{\tilde{q}_{v}-2+\epsilon_{v}}{4}\left(x_{v} f+y_{v} e\right)+\frac{q_{v}-\epsilon_{v}}{4} y_{v} f \\
G^{=}=\left(s_{v}+\frac{q-1}{2}\left(x_{v}+y_{v}\right)\right) c \\
H_{+}=s_{v} f+\frac{q-1}{2} y_{v} d+\frac{\tilde{q}_{v}-4-\epsilon_{v}}{4} y_{v} f+\frac{q_{v}-2+\epsilon_{v}}{4}\left(x_{v} f+y_{v} e\right)+\frac{\tilde{q}_{v}-\epsilon_{v}}{4} x_{v} e \\
H_{-}=s_{v} i+\frac{q-1}{2} x_{v} g+\frac{q_{v}-4-\epsilon_{v}}{4} x_{v} i+\frac{\tilde{q}_{v}-2+\epsilon_{v}}{4}\left(x_{v} h+y_{v} i\right)+\frac{q_{v}-\epsilon_{v}}{4} y_{v} h \\
I_{+}=s_{v} i+\frac{q-1}{2} x_{v} g+\frac{q_{v}-4-\epsilon_{v}}{4} x_{v} i+\frac{\tilde{q}_{v}-2+\epsilon_{v}}{4}\left(x_{v} h+y_{v} i\right)+\frac{q_{v}-\epsilon_{v}}{4} y_{v} h \\
\left.I_{-}=s_{v} f+\frac{q-1}{2} y_{v} d+\frac{\tilde{q}_{v}-4-\epsilon_{v}}{4} y_{v} f+x_{v} e\right)+\frac{\tilde{q}_{v}-\epsilon_{v}}{4} x_{v} e \\
E_{v} f+\epsilon_{v} \\
=
\end{gathered}
$$

where we have $q_{v}+\tilde{q}_{v}=2 q$, as above.

We consider moreover

$$
\begin{aligned}
& f_{v}^{s}=D+\frac{1+\epsilon_{v}}{2} E_{+}+\frac{1-\epsilon_{v}}{2} E_{-}+\frac{1+\epsilon_{v}}{2} F_{+}+\frac{1-\epsilon_{v}}{2} F_{-} \\
& f_{v}^{n}=G+\frac{1+\epsilon_{v}}{2} H_{+}+\frac{1-\epsilon_{v}}{2} H_{-}+\frac{1+\epsilon_{v}}{2} I_{+}+\frac{1-\epsilon_{v}}{2} I_{-}
\end{aligned}
$$

with $D, E_{ \pm}, F_{ \pm}, G, H_{ \pm}, I_{ \pm}$as in the definition of $E_{v}$ and we set

$$
f_{v}=f_{v}^{s}+f_{v}^{n}
$$


We have then for arbitrary $q, q_{v}, \epsilon_{v}, s_{v}, x_{v}, y_{v}, q_{w}, \epsilon_{w}, s_{w}, x_{w}, y_{w}$ with $\tilde{q}_{v}=2 q-q_{v}, \tilde{q}_{w}=$ $2 q-q_{w}$ the identity

$$
f_{v}\left(m_{v}\left(A, e_{w}(B)\right)\right)=f_{w}\left(m_{w}\left(e_{v}(A), B\right)\right)
$$

which holds for all $A, B$ and which corresponds to (9). We get thus a coloured local invariant. Observe that this identity is surprisingly general: The construction of the invariant (enumeration of invertible Schrödinger operators according to values of the Jacobi symbol on the diagonal) ensures it only for $\epsilon_{v}=\epsilon$ with $\epsilon \in\{ \pm 1\}$ such that $q \equiv \epsilon(\bmod 4)$ for $q=q_{v}=\tilde{q}_{v}=q_{w}=\tilde{q}_{w}$ an odd prime-power.

Choosing an odd prime-power $q$ and setting $q_{v}=q$ and $\epsilon_{v}=\epsilon$ where $\epsilon \in\{ \pm 1\}$ is given by $q \equiv \epsilon(\bmod 4)$, the coefficient of a monomial

$$
\prod_{v \in V(T)} u_{v}
$$

with $u_{v} \in\left\{s_{v}, x_{v}, y_{v}\right\}$ of the final result is a natural integer counting the number of invertible Schrödinger operators with diagonal coefficient at a vertex $v$ equal 0 if $u_{v}=$ $s_{v}$, respectively in the set of non-zero squares or non-squares if $u_{v}=x_{v}$ or $u_{v}=y_{v}$. Contributions from $f^{s}$ correspond to operators with determinant a non-zero square and contributions from $f^{n}$ correspond to non-square determinants.

Replacing $f_{v}$ either by $f_{v}^{s}$ or by $f_{v}^{n}$ we lose in general identity (11). It remains however valid for a few specializations. Example are:

- $\epsilon_{v}=\epsilon$ for $\epsilon \in\{ \pm 1\}, q_{v}=q$ (and $s_{v}, x_{v}, y_{v}$ arbitrary at each vertex). This case, with $\epsilon \equiv q(\bmod 4)$, counts of course Schrödinger operators with determinant a non-zero square, respectively a non-square, of $\mathbb{F}_{q}$.

- $\epsilon_{v}=\epsilon, q_{v}=q, s_{v}=\frac{1-q}{2}\left(x_{v}+y_{v}\right)$.

- $\epsilon_{v}=\epsilon, q_{v}=q, x_{v}=y_{v}=x$.

- $\epsilon_{v}=\epsilon, q_{v}=q$ and $y_{v}=-x_{v}$ at each vertex $v$ of $T$.

\section{$8 \quad$ An identity in $\mathbb{Z}\left[\mathrm{SL}_{2}\left(\mathbb{F}_{q}\right)\right]$}

We compute the coefficients of

$$
\left(\sum_{\mu \in M} \sum_{x \in \mathbb{F}_{q}}\left[\left(\begin{array}{cc}
x & \mu \\
-1 / \mu & 0
\end{array}\right)\right]\right)^{n} \in \mathbb{Z}\left[\mathrm{SL}_{2}\left(\mathbb{F}_{q}\right)\right]
$$

where $M$ is a subgroup of the multiplicative group of units $\mathbb{F}_{q}^{*}$. This allows to compute the sum over all coefficients corresponding to elements of trace 2 or of trace -2 . For $M=\{1\}$ the trivial group, these sums encode the number of Schrödinger operators of determinant zero over $\mathbb{F}_{q}$ for the $n$-cycle $C_{n}$. 
Remark 17. The factorizations

$$
\sum_{\mu \in M} \sum_{x \in \mathbb{F}_{q}}\left[\left(\begin{array}{cc}
x & \mu \\
-1 / \mu & 0
\end{array}\right)\right]=A(M) U=U A(M)
$$

where

$$
A(M)=\sum_{\mu \in M}\left[\left(\begin{array}{cc}
\mu^{-1} & 0 \\
0 & \mu
\end{array}\right)\right] \text { and } U=\sum_{x \in \mathbb{F}_{q}}\left[\left(\begin{array}{cc}
x & 1 \\
-1 & 0
\end{array}\right)\right]
$$

show that $M$ plays only a minor role in (12).

The element $A(M)$ satisfies $A(M)^{2}=m A(M)$ where $m$ is the number of elements of $M$. The element $\frac{1}{m} A(M)$ is thus a non-central idempotent (of rank $\frac{q^{3}-q}{m}$ ) in $\mathbb{Q}\left[\mathrm{SL}_{2}\left(\mathbb{F}_{q}\right)\right]$. The spectrum (with multiplicities) of the linear endomorphism of $\mathbb{Q}\left[\mathrm{SL}_{2}\right]$ defined by $X \longmapsto U X$ can also easily be recovered from our data. It consists of a subset of $\{-1,0, \pm \sqrt{q}, \pm \sqrt{-q}, q\}$, with $\pm \sqrt{-q}$ only occurring if $-1 \notin M$.

For $n \geqslant 1$ we consider the three sequences

$$
\begin{aligned}
& \alpha_{n}=\left(\begin{array}{ll}
q^{(n-1)} & (\bmod 2)
\end{array}\right) \frac{q^{2\lfloor(n-1) / 2\rfloor}-1}{q^{2}-1}-\frac{q^{\lfloor(n-1) / 2\rfloor}-1}{q-1} \\
& \beta_{n}=\left(\begin{array}{ll}
q^{(n-1)} & (\bmod 2)
\end{array}\right) \frac{q^{2\lfloor(n-1) / 2\rfloor}-1}{q^{2}-1} \\
& \gamma_{n}=\left(\begin{array}{ll}
q^{(n-1)} & (\bmod 2)
\end{array}\right) \frac{q^{2\lfloor(n-1) / 2\rfloor}-1}{q^{2}-1}-\frac{q^{\lfloor(n-1) / 2\rfloor}-1}{q-1}+\frac{q^{\lfloor(n-1) / 2\rfloor}}{m}
\end{aligned}
$$

(with $\gamma_{n}$ depending on $m$, considered as a fixed constant) with $(n-1)(\bmod 2) \in\{0,1\}$ equal 1 if $n$ is even and zero otherwise and with $\lfloor(n-1) / 2\rfloor=\frac{n-2}{2}$ for even $n$ and $\lfloor(n-1) / 2\rfloor=\frac{n-1}{2}$ for odd $n$.

For $q \geqslant 2$ a prime-power and for $m$ a natural integer dividing $q-1$ we have $\alpha_{n} \leqslant$ $\beta_{n}<\gamma_{n}$ for all $n \geqslant 1$ with equality occurring only for $\alpha_{1}=\beta_{1}=0$ and $\alpha_{2}=\beta_{2}=0$.

Given a multiplicative subgroup $M$ of $m$ elements in the unit group $\mathbb{F}_{q}^{*}$, we set $\bar{M}=$ $\mathbb{F}_{q}^{*} \backslash M$ if $-1 \in M$, respectively $\bar{M}=\mathbb{F}_{q}^{*} \backslash(+M \cup-M)$ if $-1 \notin M$. Observe that $-1 \notin M$ if and only if $m q$ is odd. Given two subsets $B, D$ of $\mathbb{F}_{q}$, we denote by $\left(\begin{array}{c}B \\ D\end{array}\right)$ the subset of all column-vectors of $\mathbb{F}_{q}^{2}$ with first coordinate in $B$ and second coordinate in $D$. We consider now the partition of all non-zero elements of $\mathbb{F}_{q}^{2}$ given by the four disjoint subsets

$$
\left(\begin{array}{c}
M \\
0
\end{array}\right),\left(\begin{array}{c}
\bar{M} \\
0
\end{array}\right),\left(\begin{array}{c}
\mathbb{F}_{q} \\
M
\end{array}\right),\left(\begin{array}{c}
\mathbb{F}_{q} \\
\bar{M}
\end{array}\right)
$$

if $-1 \in M$ (i.e. if $m q$ is even), respectively by the six disjoint subsets

$$
\left(\begin{array}{c}
+M \\
0
\end{array}\right),\left(\begin{array}{c}
-M \\
0
\end{array}\right),\left(\begin{array}{c}
\bar{M} \\
0
\end{array}\right),\left(\begin{array}{c}
\mathbb{F}_{q} \\
+M
\end{array}\right),\left(\begin{array}{c}
\mathbb{F}_{q} \\
-M
\end{array}\right),\left(\begin{array}{c}
\mathbb{F}_{q} \\
\bar{M}
\end{array}\right)
$$

if $-1 \notin M$ (i.e. if $m q$ is odd) where 0 denotes of course the singleton $\{0\} \subset \mathbb{F}_{q}$. 
For a fixed multiplicative subgroup $M$ of $m$ elements in $\mathbb{F}_{q}^{*}$ and $n \geqslant 1$, we consider the four, respectively six, rational sequences, named by the parts of the above partition of non-zero column-vectors in $\mathbb{F}_{q}^{2}$, given by table (14).

\begin{tabular}{||c||c|c|c|c||}
\hline \hline$n(\bmod 4):$ & 0 & 1 & 2 & 3 \\
\hline \hline$\left(\begin{array}{c}M \\
0\end{array}\right)_{n}$ & $\beta_{n}$ & $\gamma_{n}$ & $\beta_{n}$ & $\gamma_{n}$ \\
\hline$\left(\begin{array}{c}+M \\
0\end{array}\right)_{n}$ & $\beta_{n}$ & $\gamma_{n}$ & $\beta_{n}$ & $\alpha_{n}$ \\
\hline $\left.\begin{array}{c}-M \\
0\end{array}\right)_{n}$ & $\beta_{n}$ & $\alpha_{n}$ & $\beta_{n}$ & $\gamma_{n}$ \\
\hline$\left(\begin{array}{c}M \\
0\end{array}\right)_{n}$ & $\beta_{n}$ & $\alpha_{n}$ & $\beta_{n}$ & $\alpha_{n}$ \\
$\left(\begin{array}{c}\mathbb{F}_{q} \\
M\end{array}\right)_{n}$ & $\gamma_{n}$ & $\beta_{n}$ & $\gamma_{n}$ & $\beta_{n}$ \\
\hline $\left.\begin{array}{c}\mathbb{F}_{q} \\
+M\end{array}\right)_{n}$ & $\gamma_{n}$ & $\beta_{n}$ & $\alpha_{n}$ & $\beta_{n}$ \\
\hline$\left(\begin{array}{c}\mathbb{F}_{q} \\
-M\end{array}\right)_{n}$ & $\alpha_{n}$ & $\beta_{n}$ & $\gamma_{n}$ & $\beta_{n}$ \\
\hline$\left(\begin{array}{c}\mathbb{F}_{q} \\
\bar{M})_{n}\end{array}\right.$ & $\alpha_{n}$ & $\beta_{n}$ & $\alpha_{n}$ & $\beta_{n}$ \\
\hline \hline
\end{tabular}

Values for $\left(\begin{array}{c}M \\ 0\end{array}\right)_{n}$ and $\left(\begin{array}{c}\mathbb{F}_{q} \\ M\end{array}\right)_{n}$ apply only if $-1 \in M$ and depend only on the parity of $n$. Values for $\left(\begin{array}{c}n \\ 0\end{array}\right)_{n}$ and $\left(\frac{\mathbb{F}_{q}}{M}\right)_{n}$ apply whether or not -1 is in $M$ and depend also only on the parity of $n$. The remaining values involving $+M$ or $-M$ apply only if $-1 \notin M$ and depend on $n$ modulo 4 .

For a non-zero vector $\left(\begin{array}{l}b \\ d\end{array}\right)$ and for $n \geqslant 1$ (and for a given fixed subgroup $M \subset \mathbb{F}_{q}^{*}$ of $m$ elements) we set $\left(\begin{array}{c}b \\ d\end{array}\right)_{n}=\left(\begin{array}{c}B \\ D\end{array}\right)_{n}$ if $b \in B$ and $d \in D$ with $B, D \in\left\{M, \bar{M}, 0, \mathbb{F}_{q}\right\}$ in the case where $-1 \in M$, respectively with $B, D \in\left\{+M,-M, \bar{M}, 0, \mathbb{F}_{q}\right\}$ in the case where $-1 \notin M$. Observe that $\left(\begin{array}{c}b \\ d\end{array}\right)_{n} \in\left\{\alpha_{n}, \beta_{n}, \gamma_{n}\right\}$ for all $n \geqslant 1$. We have now the following result:

Theorem 18. For all integers $n \geqslant 1$ we have the identity

$$
\left(\sum_{\mu \in M} \sum_{x \in \mathbb{F}_{q}}\left[\left(\begin{array}{cc}
x & \mu \\
-1 / \mu & 0
\end{array}\right)\right]\right)^{n}=m^{n} \sum_{\mathrm{SL}_{2}\left(\mathbb{F}_{q}\right)}\left(\begin{array}{l}
b \\
d
\end{array}\right)_{n}\left[\left(\begin{array}{ll}
a & b \\
c & d
\end{array}\right)\right]
$$


in the group ring $\mathbb{Z}\left[\mathrm{SL}_{2}\left(\mathbb{F}_{q}\right)\right]$ where $m$ is the number of elements in a subgroup $M$ of $\mathbb{F}_{q}^{*}$ and where $\left(\begin{array}{l}b \\ d\end{array}\right)_{n}$ are as above (the last sum is of course over all $q^{3}-q$ elements $\left(\begin{array}{cc}a & b \\ c & d\end{array}\right)$ of $\mathrm{SL}_{2}\left(\mathbb{F}_{q}\right)$ ).

Remark 19. Theorem 18 can easily be adapted to $\mathrm{PSL}_{2}\left(\mathbb{F}_{q}\right)$.

The following Lemma is the main ingredient for proving Theorem 18:

Lemma 20. The three sequences $\alpha_{n}, \beta_{n}, \gamma_{n}, n \geqslant 1$ satisfy the identities

$$
\begin{aligned}
\alpha_{2 n} & =\alpha_{2 n-1}+(q-1) \beta_{2 n-1}, \\
\alpha_{2 n+1} & =q \alpha_{2 n}, \\
\beta_{n} & =q \beta_{n-1}+(n \quad(\bmod 2)), \\
& =(q-1-m) \alpha_{n-1}+\beta_{n-1}+m \gamma_{n-1}-((n-1) \quad(\bmod 2)), \\
\gamma_{2 n} & =(q-1) \beta_{2 n-1}+\gamma_{2 n-1}, \\
\gamma_{2 n+1} & =q \gamma_{2 n} .
\end{aligned}
$$

We leave the straightforward but tedious verifications to the reader.

Remark 21. The two recursive identities for $\beta_{n}$ yield

$$
(q-1-m) \alpha_{n}+m \gamma_{n}=(q-1) \beta_{n}+1
$$

which shows linear dependency of the constant sequence $1,1,1, \ldots$ from the three sequences $\alpha_{n}, \beta_{n}, \gamma_{n}$.

Proof of Theorem 18. The result holds by (14) for $n=1$ since $\alpha_{1}=\beta_{1}=0$ and $\gamma_{1}=\frac{1}{m}$.

For $n \geqslant 2$, we have the recursive formula

$$
m^{n}\left(\begin{array}{l}
b \\
d
\end{array}\right)_{n}=m^{n-1} \sum_{\mu \in M} \sum_{x \in \mathbb{F}_{q}}\left(\begin{array}{cc}
0 & -\mu \\
1 / \mu & x
\end{array}\right)\left(\begin{array}{l}
b \\
d
\end{array}\right)_{n-1}
$$

and induction implies

$$
m\left(\begin{array}{l}
b \\
d
\end{array}\right)_{n}=\sum_{\mu \in M} \sum_{x \in \mathbb{F}_{q}}\left(\begin{array}{c}
-\mu d \\
b / \mu+x d
\end{array}\right)_{n-1} .
$$

Elementary properties of finite fields imply now the recursive formulae

$$
\begin{aligned}
& \left(\begin{array}{c}
M \\
0
\end{array}\right)_{n}=q\left(\begin{array}{c}
\mathbb{F}_{q} \\
M
\end{array}\right)_{n-1} \\
& \left(\begin{array}{c}
\bar{M} \\
0
\end{array}\right)_{n}=q\left(\frac{\mathbb{F}_{q}}{\bar{M}}\right)_{n-1}
\end{aligned}
$$




$$
\begin{aligned}
& \left(\begin{array}{c}
\mathbb{F}_{q} \\
M
\end{array}\right)_{n}=\left(\begin{array}{c}
M \\
0
\end{array}\right)_{n-1}+m\left(\begin{array}{c}
\mathbb{F}_{q} \\
M
\end{array}\right)_{n-1}+(q-1-m)\left(\frac{\mathbb{F}_{q}}{M}\right)_{n-1} \\
& \left(\begin{array}{c}
\mathbb{F}_{q} \\
\bar{M}
\end{array}\right)_{n}=\left(\begin{array}{c}
\bar{M} \\
0
\end{array}\right)_{n-1}+m\left(\begin{array}{c}
\mathbb{F}_{q} \\
M
\end{array}\right)_{n-1}+(q-1-m)\left(\frac{\mathbb{F}_{q}}{M}\right)_{n-1}
\end{aligned}
$$

if $-1 \in M$ and the recursive identities

$$
\begin{aligned}
&\left(\begin{array}{c} 
\pm M \\
0
\end{array}\right)_{n}=q\left(\begin{array}{c}
\mathbb{F}_{q} \\
\pm M
\end{array}\right)_{n-1} \\
&\left(\begin{array}{c}
M \\
0
\end{array}\right)_{n}=q\left(\begin{array}{c}
\mathbb{F}_{q} \\
M
\end{array}\right)_{n-1} \\
&\left(\begin{array}{c}
\mathbb{F}_{q} \\
\pm M
\end{array}\right)_{n}=\left(\begin{array}{c}
\mp M \\
0
\end{array}\right)_{n-1}+T_{n-1} \\
&\left(\begin{array}{c}
\mathbb{F}_{q} \\
\bar{M}
\end{array}\right)_{n}=\left(\begin{array}{c}
\bar{M} \\
0
\end{array}\right)_{n-1}+T_{n-1}
\end{aligned}
$$

where

$$
T_{n-1}=m\left(\begin{array}{c}
\mathbb{F}_{q} \\
+M
\end{array}\right)_{n-1}+m\left(\begin{array}{c}
\mathbb{F}_{q} \\
-M
\end{array}\right)_{n-1}+(q-1-2 m)\left(\frac{\mathbb{F}_{q}}{M}\right)_{n-1}
$$

if $-1 \notin M$. Replacing all expressions by their values given by (14) we check that all these expressions boil down to equalities of Lemma 20.

Remark 22. Spectral calculus gives a different proof of Theorem 18.

\subsection{Traces}

We denote by $m^{n} S_{\tau}(n)$ the sum of all coefficients in (12) corresponding to elements of trace $\tau \in \mathbb{F}_{q}$. We are only interested in the values of $S_{2}(n)$ and $S_{-2}(n)$. Observe that $\mathrm{SL}_{2}\left(\mathbb{F}_{q}\right)$ contains exactly $q^{2}$ elements of trace 2 : The identity and all (other) $q^{2}-1$ unipotent elements. Multiplication by -1 induces of course a bijection between elements of trace $\tau$

\begin{tabular}{|c|c|c|}
\hline & $n \equiv 0 \quad(\bmod 4)$ & $n \equiv 2 \quad(\bmod 4)$ \\
\hline$\kappa(2, n)$ & 1 & 0 \\
\hline$\kappa(-2, n)$ & 0 & 1 \\
\hline
\end{tabular}
and elements of trace $-\tau$.

We have the following result:

Proposition 23. If $n$ is even we have

$$
\begin{aligned}
S_{ \pm 2}(n)= & q^{3} \frac{q^{n-2}-1}{q^{2}-1}-\left(q^{2}-q+1\right) \frac{q^{(n-2) / 2}-1}{q-1} \\
& +\frac{m(q-1)+q \kappa( \pm 2, n)}{m} q^{(n-2) / 2}
\end{aligned}
$$

with $\kappa( \pm 2, n)=1$ if $-1 \in M$ and with $\kappa( \pm 2, n)$ given by 
if $-1 \notin M$.

If $n$ is odd, we have

$$
S_{2}(n)=S_{-2}(n)=\frac{q^{n+1}-1}{q^{2}-1}
$$

independently of $M$.

Proof. Counting the number of matrices of trace 2, respectively -2 , in every possible class we get

$$
\begin{aligned}
S_{ \pm 2}(n)= & m\left(\begin{array}{c}
M \\
0
\end{array}\right)_{n}+(q-1-m)\left(\begin{array}{c}
\bar{M} \\
0
\end{array}\right)_{n} \\
& +(m(q-1)+q)\left(\begin{array}{c}
\mathbb{F}_{q} \\
M
\end{array}\right)_{n}+(q-1-m)(q-1)\left(\frac{\mathbb{F}_{q}}{M}\right)_{n} .
\end{aligned}
$$

if $-1 \in M$ and

$$
\begin{aligned}
S_{ \pm 2}(n)= & m\left(\begin{array}{c}
+M \\
0
\end{array}\right)_{n}+m\left(\begin{array}{c}
-M \\
0
\end{array}\right)_{n}+(q-1-2 m)\left(\begin{array}{c}
\bar{M} \\
0
\end{array}\right)_{n} \\
& +(m(q-1)+q)\left(\begin{array}{c}
\mathbb{F}_{q} \\
\pm M
\end{array}\right)_{n}+m(q-1)\left(\begin{array}{c}
\mathbb{F}_{q} \\
\mp M
\end{array}\right)_{n} \\
& +(q-1-2 m)(q-1)\left(\frac{\mathbb{F}_{q}}{M}\right)_{n} .
\end{aligned}
$$

if $-1 \notin M$.

If $-1 \in M$, the above expression for $S_{ \pm 2}(n)$ amounts to

$$
\begin{aligned}
& (q-1) \beta_{n}+(m(q-1)+q) \gamma_{n}+(q-1-m)(q-1) \alpha_{n} \\
= & q^{3} \frac{q^{n-2}-1}{q^{2}-1}-\left(q^{2}-q+1\right) \frac{q^{(n-2) / 2}-1}{q-1}+(m(q-1)+q) \frac{q^{(n-2) / 2}}{m}
\end{aligned}
$$

if $n$ is even and to

$$
m \gamma_{n}+(q-1-m) \alpha_{n}+\left(q^{2}-q+1\right) \beta_{n}=\frac{q^{n+1}-1}{q^{2}-1}
$$

if $n$ is odd.

If $-1 \notin M$ and $n$ even the value of $S_{\tau}(n)$ with $\tau \in\{ \pm 2\}$ equals

$$
(q-1) \beta_{n}+(q-1-m)(q-1) \alpha_{n}+m(q-1) \gamma_{n}+q \eta_{n}(\tau)
$$

with $\eta_{n}(\tau)$ given by

\begin{tabular}{||c||cc|cc||}
\hline \hline & $n \equiv 0$ & $(\bmod 4)$ & $n \equiv 2 \quad(\bmod 4)$ \\
\hline \hline$\eta_{n}(2)=$ & $\gamma_{n}$ & $\alpha_{n}$ \\
\hline$\eta_{n}(-2)=$ & $\alpha_{n}$ & $\gamma_{n}$. \\
\hline \hline
\end{tabular}


For $\eta_{n}(\tau)=\alpha_{n}$ we have

$$
q^{3} \frac{q^{n-2}-1}{q^{2}-1}-\left(q^{2}-q+1\right) \frac{q^{(n-2) / 2}-1}{q-1}+(q-1) q^{(n-2) / 2}
$$

and for $\eta_{n}(\tau)=\gamma_{n}$ we get

$$
q^{3} \frac{q^{n-2}-1}{q^{2}-1}-\left(q^{2}-q+1\right) \frac{q^{(n-2) / 2}-1}{q-1}+(m(q-1)+q) \frac{q^{(n-2) / 2}}{m} .
$$

For $-1 \notin M$ and $n$ odd, the common value $S_{2}(n)=S_{-2}(n)$ is given by

$$
m\left(\alpha_{n}+\gamma_{n}\right)+(q-1-2 m) \alpha_{n}+\left(q^{2}-q+1\right) \beta_{n}=\frac{q^{n+1}-1}{q^{2}-1} .
$$

This ends the proof.

\section{Proof of Theorem 2}

Proof. We denote by $I_{n}$ the graph having vertices $1, \ldots, n$. Consecutive integers represent adjacent vertices. For $q$ a fixed prime-power and $n \geqslant 1$, we write $\nu\left(\begin{array}{ll}a & b \\ c & d\end{array}\right)_{n}$ for the number of Schrödinger operators $M$ over $\mathbb{F}_{q}$ for $I_{n}$ such that

$$
\begin{array}{ll}
a=\operatorname{det}(M) & b=\operatorname{det}(M(n ; n)) \\
c=-\operatorname{det}(M(1 ; 1)) & d=-\operatorname{det}(M(1, n ; 1, n))
\end{array}
$$

where $M(i ; j)$ respectively $M\left(i_{1}, i_{2} ; j_{1}, j_{2}\right)$ denotes the submatrix of $M$ obtained by deleting line $(\mathrm{s}) i_{*}$ and row $(\mathrm{s}) j_{*}$.

Initial values for $\nu\left(\begin{array}{ll}a & b \\ c & d\end{array}\right)_{1}$ are given by $\nu\left(\begin{array}{cc}x & 1 \\ -1 & 0\end{array}\right)_{1}=1$ and $\nu\left(\begin{array}{ll}a & b \\ c & d\end{array}\right)_{1}=0$ if $(b, c, d) \neq(1,-1,0)$. Expanding the determinant of a Schrödinger operator for $I_{n+1}$ with a first diagonal coefficient $x$ along the first row shows the recursion

$$
\nu\left(\begin{array}{ll}
a & b \\
c & d
\end{array}\right)_{n+1}=\sum_{x \in \mathbb{F}_{q}} \nu\left(\begin{array}{cc}
-c & -d \\
a+x c & b+x d
\end{array}\right)_{n} .
$$

The matrix identity

$$
\left(\begin{array}{ll}
a & b \\
c & d
\end{array}\right)=\left(\begin{array}{cc}
x & 1 \\
-1 & 0
\end{array}\right)\left(\begin{array}{cc}
-c & -d \\
a+x c & b+x d
\end{array}\right)
$$

implies now the identity

$$
\left(\sum_{x \in \mathbb{F}_{q}}\left[\left(\begin{array}{cc}
x & 1 \\
-1 & 0
\end{array}\right)\right]\right)^{n}=\sum_{\mathrm{SL}_{2}\left(\mathbb{F}_{q}\right)} \nu\left(\begin{array}{ll}
a & b \\
c & d
\end{array}\right)_{n}\left[\left(\begin{array}{ll}
a & b \\
c & d
\end{array}\right)\right]
$$


in the group-ring $\mathbb{Z}\left[\mathrm{SL}_{2}\left(\mathbb{F}_{q}\right)\right]$. This correspond of course to the case $M=1$ in (12) or in Theorem 18 , with $-1 \notin M$ except if $\mathbb{F}_{q}$ is of characteristic 2 .

Given a Schrödinger operator $M$ for $I_{n}$ we denote by $\tilde{M}$ the Schrödinger operator of the $n$-cycle (obtained by joining the first and last vertex of $I_{n}$ with an additional edge) with the same diagonal coefficients. Thus, $\tilde{M}$ is obtained from $M$ by adding two non-zero coefficients 1 at the upper-right and lower-left corner of $M$. Denoting by $x_{1}$ the first diagonal coefficient of $M$ or $\tilde{M}$ and expanding the determinant of $\tilde{M}$ along the first row and perhaps subsequently along the first column, we get

$$
\begin{aligned}
\operatorname{det}(\tilde{M})= & x_{1} \operatorname{det}(M(1 ; 1))-\operatorname{det}(M(1,2 ; 1,2)) \\
& -(-1)^{n}(\operatorname{det}(M(1, n ; 1,2))+\operatorname{det}(M(1,2 ; 1, n))) \\
& -\operatorname{det}(M(1, n ; 1, n)) .
\end{aligned}
$$

The identities

$$
\begin{gathered}
x_{1} \operatorname{det}(M(1 ; 1))-\operatorname{det}(M(1,2 ; 1,2))=\operatorname{det}(M)=a, \\
\operatorname{det}(M(1, n ; 1,2))=\operatorname{det}(M(1,2 ; 1, n))=1, \\
-\operatorname{det}(M(1, n ; 1, n))=c
\end{gathered}
$$

with $\left(\begin{array}{ll}a & b \\ c & d\end{array}\right)$ the matrix associated to $M$ as above yield

$$
\operatorname{det}(\tilde{M})=a+c-2(-1)^{n}
$$

The number of non-invertible Schrödinger operators for $C_{n}$ is thus the total sum $S_{2(-1)^{n}}(n)$ of coefficients associated to matrices of trace $2(-1)^{n}$ in

$$
\left(\sum_{x \in \mathbb{F}_{q}}\left[\left(\begin{array}{cc}
x & 1 \\
-1 & 0
\end{array}\right)\right]\right)^{n} \text {. }
$$

This shows that $q^{n}-S_{2(-1)^{n}}(n)$ is the number of invertible Schrödinger operators for $C_{n}$. Proposition 23 with $m=1$ and $M=\{1\}$ the trivial subgroup of $\mathbb{F}_{q}$ gives the values for $S_{2(-1)^{n}}$. Observe that $-1 \in M$ if $q$ is a power of 2 and $-1 \notin M$ if $q$ is an odd prime-power. The easy identities

$$
\begin{aligned}
& q^{2 n}-\frac{\left(1-q^{2 n}\right)\left(1-q^{2 n+1}\right)}{1-q^{2}} \\
= & q^{3} \frac{q^{4 n-2}-1}{q^{2}-1}-\left(q^{2}-q+1\right) \frac{q^{2 n-1}-1}{q-1}+(2 q-1) q^{2 n-1}
\end{aligned}
$$

and

$$
-\frac{\left(1-q^{2 n+1}\right)\left(1-q^{2 n+2}\right)}{1-q^{2}}
$$




$$
=q^{3} \frac{q^{4 n}-1}{q^{2}-1}-\left(q^{2}-q+1\right) \frac{q^{2 n}-1}{q-1}+(q-1) q^{2 n}
$$

end the proof.

Remark 24. Since $S_{2}(n)=S_{-2}(n)$ for odd $n$, the number of invertible Schrödinger operators for $C_{n}$ is also given by $q^{n}-S_{2}(n)$.

\section{Proof for complete graphs}

\subsection{Simple stars}

The number of invertible Schrödinger operators of simple stars (finite graphs with at most one non-leaf) is a crucial ingredient for proving Theorem 3.

We denote in this subsection by $R_{n}$ the rooted graph consisting of a central root adjacent to $n-1$ leaves. Writing $S_{r}\left(R_{n}\right)=\left(a_{n}, b_{n}\right)$ with $S_{r}\left(R_{n}\right)$ defined as in Section 3, we have

$$
S_{R_{n}}=\left((q-1)^{n-1},(n-1)(q-1)^{n-2}\right)
$$

as can be checked using

$$
\begin{aligned}
\left(a_{n+1}, b_{n+1}\right) & =m\left(\left(a_{n}, b_{n}\right), e(v)\right)=m\left(\left(a_{n}, b_{n}\right),(q-1,1)\right) \\
& =\left((q-1) a_{n}, a_{n}+(q-1) b_{n}\right)
\end{aligned}
$$

We have thus

$$
S_{*_{n}}=f\left(a_{n}, b_{n}\right)=\left(q^{2}+(n-3) q+1\right)(q-1)^{n-2}
$$

for the non-rooted star $*_{n}$ underlying $R_{n}$ given by a central vertex of degree $n-1$ surrounded by $n-1$ leaves.

\subsection{Proof of Theorem 3}

We consider an invertible Schrödinger operator $M$ for the star $*_{n}$ with a central vertex of degree $n-1$ surrounded by $n-1$ leaves. If the diagonal coefficient $\lambda$ of the central vertex is different from -1, we get an invertible Schrödinger operator of the complete graph $K_{n}$ on $n$ vertices by adding the first row, corresponding to the central vertex of $*_{n}$, of $M$ to all other rows and by dividing the first column of the resulting matrix by $1+\lambda$. This construction can be reversed, as easily seen on the following illustration:

$$
\left(\begin{array}{ccccc}
\lambda & 1 & 1 & \ldots & 1 \\
1 & a_{2} & 0 & \ldots & 0 \\
1 & 0 & a_{3} & \ldots & 0 \\
\vdots & & & \ddots & \vdots \\
1 & 0 & 0 & \ldots & 0 \\
1 & 0 & 0 & \ldots & a_{n}
\end{array}\right) \leftrightarrow\left(\begin{array}{cccccc}
\lambda & 1 & 1 & \ldots & 1 \\
1+\lambda & 1+a_{2} & 1 & \ldots & 1 \\
1+\lambda & 1 & 1+a_{3} & \ldots & 1 \\
\vdots & & & \ddots & \vdots \\
1+\lambda & 1 & 1 & \ldots & 1+a_{n}
\end{array}\right)
$$




$$
\leftrightarrow\left(\begin{array}{cccccc}
\mu & 1 & 1 & \ldots & 1 & 1 \\
1 & 1+a_{2} & 1 & \ldots & 1 & 1 \\
1 & 1 & 1+a_{3} & \ldots & 1 & 1 \\
\vdots & & & \ddots & & \vdots \\
1 & 1 & 1 & \ldots & a_{n-1} & 1 \\
1 & 1 & 1 & \ldots & 1 & 1+a_{n}
\end{array}\right)
$$

Every invertible Schrödinger operator of $K_{n}$ with first diagonal coefficient $\mu=\lambda /(1+\lambda)$ different from 1 is of this form by taking $\lambda=\frac{\mu}{1-\mu}$.

Schrödinger operators of $K_{n}$ with first coefficient 1 are invertible if and only if they have a first diagonal coefficient equal to 1 and all $n-1$ remaining diagonal coefficients different from 1 . There are thus $(q-1)^{n-1}$ such matrices.

The number $\kappa_{n}$ of invertible Schrödinger operators for the complete graph $K_{n}$ (over a fixed finite field $\mathbb{F}_{q}$ ) is thus given by

$$
\kappa_{n}=S_{*_{n}}(q)+(q-1)^{n-1}-s_{n}
$$

where $S_{*_{n}}$ is given by (18) and where $s_{n}$ denotes the number of invertible Schrödinger operators of $*_{n}$ with -1 as the diagonal entry corresponding to the central vertex of the simple star $*_{n}$ consisting of a central vertex of degree $n-1$ adjacent to $n-1$ leaves.

The number $s_{n}$ can be computed as follows: First observe that an invertible Schrödinger operator of $*_{n}$ has at most a unique diagonal coefficient which is zero. The contribution of such matrices to $s_{n}$, given by

$$
(n-1)(q-1)^{n-2},
$$

is easy to establish.

Matrices contributing to $s_{n}$ having only non-zero diagonal entries are in bijection with solutions $\left(b_{2}, \ldots, b_{n}\right) \in\left(\mathbb{F}_{q}^{*}\right)^{n-1}$ of

$$
-b_{2} \cdots b_{n}\left(1+\frac{1}{b_{2}}+\cdots+\frac{1}{b_{n}}\right) \neq 0 .
$$

We denote by $\tilde{s}_{n}$ the number of such solutions $\left(b_{2}, \ldots, b_{n}\right) \in\left(\mathbb{F}_{q}^{*}\right)^{n-1}$.

Choosing an arbitrary non-zero element $x_{1} \in \mathbb{F}_{q}^{*}$ and setting $x_{2}=\frac{x_{1}}{b_{2}}, \ldots, x_{n}=\frac{x_{1}}{b_{n}}$ we have $(q-1) \tilde{s}_{n}=\beta_{n}$ where $\beta_{n}$ counts the number of solutions $\left(x_{1}, \ldots, x_{n}\right) \in\left(\mathbb{F}_{q}^{*}\right)^{n}$ of the inequality $\sum_{i=1}^{n} x_{i} \neq 0$. We denote similarily by $\alpha_{n}$ the number of solutions $\left(x_{1}, \ldots, x_{n}\right) \in\left(\mathbb{F}_{q}^{*}\right)^{n}$ of the equality $\sum_{i=1}^{n} x_{i}=0$.

We have $\alpha_{0}=1, \beta_{0}=0$ and the recursive formulae

$$
\begin{aligned}
\alpha_{n} & =\beta_{n-1}, \\
\beta_{n} & =(q-1) \alpha_{n-1}+(q-2) \beta_{n-1} .
\end{aligned}
$$

Lemma 25. We have

$$
\beta_{n}=\frac{(q-1)^{n+1}+(-1)^{n+1}(q-1)}{q} .
$$


Proof. The sequence $\beta_{n}$ has the recursive definition $\beta_{0}=0, \beta_{1}=q-1$ and $\beta_{n}=(q-$ 2) $\beta_{n-1}+(q-1) \beta_{n-2}$ for $n \geqslant 2$. An induction on $n$ based on the trivial identity

$$
\begin{aligned}
(q-1)^{n+1}+(-1)^{n+1}(q-1)= & (q-2)\left((q-1)^{n}+(-1)^{n}(q-1)\right) \\
& +(q-1)\left((q-1)^{n-1}+(-1)^{n-1}(q-1)\right)
\end{aligned}
$$

ends the proof.

Remark 26. Lemma 25 follows also easily from the fact that the characteristic polynomial $X^{2}-(q-2) X-(q-1)$ of the recursion defining $\beta_{n}$ has roots $q-1$ and -1 .

$(20)$ and the equality $(q-1) \tilde{s}_{n}=\beta_{n}$ already mentionned imply $s_{n}=(n-1)(q-$ $1)^{n-2}+\frac{1}{q-1} \beta_{n}$. Lemma 25 shows thus that

$$
s_{n}=(n-1)(q-1)^{n-2}+\frac{(q-1)^{n}-(-1)^{n}}{q} .
$$

Identities (18), (19) and (21), taken together, show that $K_{n}$ has

$$
\begin{aligned}
& \left(q^{2}+(n-3) q+1\right)(q-1)^{n-2}+(q-1)^{n-1} \\
& -\left((n-1)(q-1)^{n-2}+\frac{(q-1)^{n}-(-1)^{n}}{q}\right) \\
= & n(q-1)^{n-1}+\frac{(q-1)^{n-2}\left(q^{3}-2 q^{2}+q-(q-1)^{2}\right)+(-1)^{n}}{q} \\
= & n(q-1)^{n-1}+\frac{(q-1)^{n+1}+(-1)^{n}}{q}
\end{aligned}
$$

invertible Schrödinger operators over $\mathbb{F}_{q}$.

\section{Final remarks}

\subsection{Generalizations}

It is of course possible to define Schrödinger operators for arbitrary (perhaps oriented) simple graphs and to count invertible Schrödinger operators over finite fields. I ignore if there is an efficient way for computing the corresponding numbers.

Enumerating for example all Schrödinger operators over $\mathbb{F}_{3}$ and $\mathbb{F}_{5}$ for the Petersen graph (obtained by identifying opposite points of the 1 -skeleton of the dodecahedron) we get $q^{10}-q^{9}+q^{8}$ invertible Schrödinger operators in both cases. This formula fails however for $\mathbb{F}_{2}$ for which no invertible Schrödinger operators exists.

A second notion, closely related to Schrödinger operators and used for example in Proposition 9, is to look at the set of all invertible matrices with off-diagonal support defining a given graph (diagonal elements are arbitrary). In the case of unoriented graphs, one can moreover require matrices to be symmetric.

For trees, both definitions are essentially identical (up to a factor $\left.(q-1)^{*}\right)$. 


\subsection{Counting points over finite fields for algebraic varieties over $\overline{\mathbb{Q}}$}

Our main problem, counting invertible Schrödinger operators, is of course a particular case of counting points over finite fields on algebraic varieties defined over $\mathbb{Z}$ (or more generally over $\overline{\mathbb{Q}})$. Such problems are in general difficult, see e.g. the monograph [4] devoted to such questions.

The main problem is of course the question if the behaviour of invertible (or equivalently, non-invertible) Schrödinger operators of graphs is simpler. All our examples give rise to polynomials (depending perhaps on the parity of the characteristic) enumerating invertible Schrödinger operators over finite fields. Does this fail for some finite graph $G$ or do there always exist polynomials depending on $q\left(\bmod N_{G}\right)$ for some natural integer $N_{G}$ evaluating to the number of invertible Schrödinger operators over $\mathbb{F}_{q}$ for $G$ ?

\section{Acknowledgements}

I thank E. Peyre and P. De la Harpe for interesting discussions and remarks.

\section{References}

[1] A. Cayley. On the Theory of the Analytical Forms called Trees. Philosophical Magazine, XIII:172-176, 1857.

[2] J. McKee, C. Smyth. Salem numbers, Pisot numbers, Mahler measure, and graphs. Experiment. Math. 14(2):211-229, 2005.

[3] OEIS Foundation Inc. (2011), The On-Line Encyclopedia of Integer Sequences, http://oeis.org.

[4] J-P. Serre. Lectures on Nx(p), CRC Press, London-New York, 2012. 